\title{
Validez del supuesto de neutralidad del horizonte de tiempo en el CAPM y la metodología del rango reescalado: aplicación a Colombia
}

Karen Juliet Leiton Rodríguez

kleitoro@banrep.gov.co

* Profesional especializado en deuda pública. Departamento de Operaciones y Desarrollo de Mercados. Subgerencia Monetaria de Reservas. Banco de la República. Profesional en economía y profesional en finanzas. Universidad del Rosario. 



\section{Introducción}

El Capital Asset Pricing Model (CAPM), desarrollado por Treynor, Sharpe, Lintner y Mossin en los años 60, es uno de los pilares de la teoría financiera moderna y fue el primero en introducir la noción de riesgo dentro de un modelo de equilibrio general para la valoración de activos financieros basándose en las características específicas de riesgo de cada activo (Litterman, 2003). Este modelo, estándar en los programas de enseñanza de finanzas corporativas, además de ser enseñado en la academia, es ampliamente utilizado en la práctica. Mandelbrot y Hudson (2004) mencionan que una encuesta realizada por dos estudiantes de la Universidad de Duke a 392 gerentes financieros de compañías pertenecientes al U.S Fortune 500, mostraba que en 1999 el $73.5 \%$ de los encuestados estimaban el costo del capital con el CAPM. De forma similar, en Europa, en 2001 el porcentaje ascendió a 77\%.

Sin embargo, pese a su trascendencia y difusión, la literatura contiene numerosos trabajos que muestran la invalidez de los supuestos del CAPM. No obstante, de acuerdo con Shojai y Feiger (2009), dicha literatura se concentra en los problemas que pueden ser resueltos analíticamente ${ }^{1}$ y no en aquellos a los que realmente se enfrenta el mercado de capitales.

Uno de esos supuestos que la teoría ha dejado de lado, y que la práctica ha ignorado, es que el CAPM fue creado como un modelo de un solo período, por lo que i) todos los agentes tienen el mismo horizonte de inversión y ii) existe neutralidad en el horizonte de inversión. Este supuesto recae sobre la independencia serial de los retornos, que es uno de los supuestos fundamentales del movimiento browniano.

Autores como Holton (1992) y Peters (1989) han resaltado la importancia del horizonte de inversión en el estudio de las series financieras, valoración de activos y administración del riesgo debido a que el riesgo puede variar con el horizonte de inversión; como en los casos en los que no hay consistencia temporal de la volatilidad, es decir, cuando la información contenida en la volatilidad de un día puede no ser igual que la de un año. En consecuencia, el movimiento browniano no sería un proceso adecuado para describir la trayectoria de los activos.

La razón por la que no se cumple la consistencia temporal de la volatilidad es consecuencia de un fenómeno encontrado inicialmente en las ciencias naturales

1 Por ejemplo, el modelo beta-cero de Black (1972) que muestra que el CAPM puede seguir siendo válido sin un activo libre de riesgo, para lo cual Black incluye un portafolio con un beta igual a cero. Otro ejemplo es la posibilidad de incorporar impuestos Brennan (1970). 
por el físico H.E. Hurst, quien anotó que el comportamiento del flujo de agua del río Nilo presentaba dependencia o memoria de largo plazo. Según la evidencia, años de alto (bajo) flujo eran seguidos por otros de alto (bajo) flujo de agua, de manera que un proceso de memoria de corto plazo no podía describir este fenómeno. Posteriormente, este comportamiento también fue encontrado por Mandelbrot (1972) en las series financieras.

La existencia de memoria o dependencia serial de largo plazo, en las series financieras, implica que los retornos de un activo hoy pueden tener incidencia sobre los retornos futuros, incluso más allá del corto plazo. Este fenómeno está asociado a la existencia del "sentimiento del mercado", que puede hacer que una tendencia persista en el mercado aun cuando no hay un evento en desarrollo o nuevas noticias que incorporar (Peters, 1989).

Holton (1992), León y Vivas (2010) y León y Reveiz (2010) muestran que la principal consecuencia práctica de la dependencia serial de largo plazo de los retornos es que su presencia viola los supuestos del movimiento browniano, lo cual tiene implicaciones teóricas y prácticas. En el campo teórico, según Peters (1989) y Lo (1991), la existencia de memoria de largo plazo invalida la hipótesis de los mercados eficientes (EMH). En la práctica, la regla de la raíz cuadrada del tiempo para escalar la volatilidad a otros plazos pierde validez ante la ausencia del supuesto de movimiento browniano. En ambos casos, el resultado de muchos modelos para la optimización de portafolio y el manejo de riesgo puede cambiar al incorporar el efecto de la memoria de largo plazo de las series financieras.

Con el fin de incorporar este efecto, León y Vivas (2010) y León y Reveiz (2010) retoman la literatura sobre la memoria de largo plazo y utilizan el análisis del rango reescalado y el exponente de Hurst como una metodología para medir la dependencia de largo plazo en las series financieras.

De acuerdo con los hallazgos de Peters (1989 y 1992), Mandelbrot (1972), León y Reveiz (2010), Ambrose et ál. (1993) y Bilel y Nadhem (2009) para series de tiempo financieras de mercados desarrollados, y de León y Vivas (2010) para el mercado colombiano, existen activos cuyos retornos exhiben memoria de largo plazo, lo cual invalidaría uno de los principales supuestos del CAPM: la neutralidad en el horizonte de inversión.

En atención a dicha evidencia, y a partir de los hallazgos generales de León y Vivas (2010) respecto de la dependencia de largo plazo de las series de tiempo financieras y sus principales implicaciones, este documento se ocupará de extender el análisis al uso del supuesto de neutralidad del horizonte de inversión en el 
CAPM, así como estimar el efecto cuantitativo de la existencia de dependencia de largo plazo en este modelo.

Es importante destacar que este trabajo no se ocupa de los problemas de autocorrelación de corto plazo en las series de tiempo, sino de la dependencia de largo plazo, aquella en que las observaciones pueden encontrarse significativamente distantes entre sí. Dado que el enfoque escogido no es de tipo econométrico clásico, sino basado en algunos desarrollos básicos de la geofísica, este trabajo no muestra pruebas ni evidencia de estacionariedad de las series de tiempo, ni del exponente de Hurst, sino está enfocado en la manera en que la volatilidad de las series cambia a través del tiempo. Por lo anterior, la escogencia de este enfoque obedece a la evidencia (Mandelbrot, 1972; Sornette, 2003) según la cual existen dificultades en la utilización de la autocorrelación y otros métodos econométricos tradicionales en series de tiempo no normales.

\section{Movimiento browniano y movimiento browniano fractal ${ }^{2}$}

Los procesos aleatorios juegan un papel importante en una gran parte de fenómenos naturales. En 1828 el botánico Robert Brown publicó un documento sobre el movimiento de las partículas del polen, que observadas bajo un microscopio, parecían moverse de forma continua y errática, de tal forma que su movimiento pasado parecía no afectar su dirección futura; los trabajos de Brown dan el nombre a lo que se conoce como movimiento browniano.

En 1905 Albert Einstein proporcionó una explicación sobre cómo moléculas pequeñas con movimiento aleatorio podrían afectar el movimiento de partículas lo suficientemente grandes como para ser observadas bajo el microscopio. Einstein utilizó métodos estadísticos para mostrar que una partícula suspendida podía ser conducida en diferentes direcciones por el efecto combinado del impacto de muchas moléculas. Pero Einstein no sabía acerca del trabajo experimental sobre el movimiento browniano. Después del trabajo de muchos físicos, en 1920 el matemático Norbert Wiener transformó la caminata aleatoria (discreta) a un modelo matemático continuo de movimiento browniano.

No obstante, la primera persona en descubrir la conexión matemática entre la caminata aleatoria y el movimiento browniano fue el matemático Louis Bachelier,

2 Esta sección se basó extensivamente en el trabajo de Peterson (1998); las citas se omitieron para facilitar la lectura.

PP. $203-245 \cdot N .^{\circ} 6 / 2011$ 
quien en 1900 anticipó muchos de los descubrimientos hechos más tarde sobre este movimiento. Bachelier, en su tesis doctoral escribió sobre las fluctuaciones aleatorias de los precios de las acciones y bonos en la bolsa de París. Sin embargo, como estaba alejado del campo de la física, y en finanzas el tema aún no se había investigado, sus ideas no tuvieron difusión inicialmente.

En su modelo para valorar opciones, Bachelier argumentó que graficar el precio pasado de una acción para predecir su futuro no era muy útil, ya que los cambios de precios sucesivos eran independientes y cualquier variación del precio correspondía a la incorporación de nueva información. Según Bachelier, alguna información es predecible y otra no, y cuando es predecible y el mercado funciona efectivamente, ya se encuentra incorporada en el precio de la acción.

A mediados de la década de 1950 Paul Samuelson, quien investigaba sobre valoración de opciones, retomó la tesis de Bachelier. Samuelson descubrió dos defectos en el movimiento browniano de Bachelier. Primero, que el valor de las opciones de largo plazo puede llegar a exceder el precio del subyacente y, segundo, que el precio de los activos puede llegar a ser negativo. Por lo anterior, Samuelson reemplazó el llamado movimiento browniano aritmético, en el cual el proceso aleatorio describe la dinámica de los precios, por el movimiento browniano geométrico, el cual describe la dinámica de los retornos logarítmicos de los precios de los activos. El movimiento browniano geométrico es el supuesto más utilizado al referirse a la dinámica de los activos financieros, al cual este documento se referirá en adelante como movimiento browniano (MB) (León 2009).

En la teoría financiera, las matemáticas aplicadas comenzaron a ser uno de los campos más activos debido a la necesidad de hallar precios justos para los activos, y de entender cómo esos precios cambian junto con otros precios y bienes. Muchas teorías de las finanzas modernas han sido fundamentadas bajo el supuesto del MB, entre ellas, la teoría de portafolio de Markowitz, el CAPM, la hipótesis de los mercados eficientes y el modelo de Black y Scholes.

El precio de un activo puede describirse mediante un MB cuando los cambios en los precios son (Mandelbrot y Hudson, 2004):

i) Continuos: no existen saltos.

ii) Independientes: implica que el cambio en el precio de hoy no está relacionado con los cambios pasados, lo que significa que cualquier información sobre el precio futuro está contenida en el precio de hoy, por lo que no es necesario observar la historia. 
iii) Estacionarios: significa que el proceso que genera los cambios de los precios es el mismo a través del tiempo. Es decir, la media y la varianza son constantes en el tiempo.

iv) Se distribuyen como una normal.

Al evaluar estos supuestos la literatura ha encontrado evidencia que cuestiona la validez del supuesto de MB para describir la dinámica de las variables financieras. León (2009), basado en la aplicación del modelo de difusión con saltos de Merton (1976), mostró evidencia de la presencia de saltos en las distribuciones de los retornos de activos colombianos, lo que contradice el supuesto de continuidad: Sornette (2003) documentó la presencia de caídas sostenidas (drawdowns) en el precio de los activos, las cuales van en contra del supuesto de normalidad e independencia de las series de tiempo. Por su parte, Engle (1982) y Bollerslev (1986) encontraron evidencia de agrupamiento en la volatilidad o volatility clustering en los retornos financieros. Esto muestra que la volatilidad cambia en el tiempo y que puede haber una correlación de los retornos pasados con los futuros, contradiciendo los supuestos de estacionariedad e independencia. Respecto del supuesto de normalidad, entre muchos autores que han evaluado la validez de los supuestos del MB, Mitchell (1915) y Mills (1927) fueron los primeros que encontraron que los retornos de los precios -de los commodities- no tienen una distribución normal.

De acuerdo con León y Vivas (2010), aunque la invalidez de esos supuestos ha tenido como consecuencia el desarrollo de modelos que capturan de forma más rigurosa la realidad ${ }^{3}$, aún persisten metodologías que recaen en el MB y que aún son implementadas en la práctica para tomar decisiones de inversión.

Según León y Reveiz (2011) el supuesto más importante del MB es el de independencia serial debido a que:

i) Si los retornos no se distribuyen como una normal, pero son independientes, entonces por el teorema del límite central, los retornos convergen en una distribución normal.

ii) El supuesto de independencia permite utilizar la regla de la raíz de tiempo para escalar la volatilidad a diferentes plazos. Sornette (2003) afirma que esta regla es la predicción más importante del MB.

3 Como modelos GARCH, ARMA, de difusión con saltos y distribuciones t-student sesgadas. 
La regla de la raíz de tiempo para escalar la volatilidad establece que, debido a que en un MB la varianza es proporcional al intervalo de tiempo, entonces la desviación estándar también es proporcional a la raíz de ese intervalo. De esta manera, según los supuestos del MB, es correcto afirmar que la desviación estándar, estimada sobre una serie de frecuencia $n$ puede escalarse a una frecuencia $d$ de la forma (1), lo cual es equivalente en términos de varianza a (2).

$$
\begin{gathered}
\sigma_{d}=\sqrt{d \sigma_{n}} \\
\sigma_{d}^{2}=\sqrt{d \sigma_{n}^{2}}
\end{gathered}
$$

Esta regla supone que existe consistencia temporal en la volatilidad; es decir, que la información contenida en la volatilidad estimada con una frecuencia $n$ es exactamente la misma a la de frecuencia $d$, o a la de cualquier otra frecuencia. La justificación de esta regla recae en que los activos financieros siguen un MB, en el que los retornos son independientes e idénticamente distribuidos como una normal. Al invalidarse ese supuesto, la volatilidad de corto plazo puede contener información diferente que la de largo plazo y, por lo tanto, el horizonte de tiempo comienza a ser otra variable importante en la medición del riesgo (Holton, 1992).

Según Peters (1989), la muy difundida aleatoriedad de los retornos en los mercados de bonos y acciones oculta una estructura fractal subyacente. Esto es, que hay patrones o tendencias en los retornos de los activos del mercado de capitales que persisten en el tiempo.

Esta tendencia o dependencia de las series financieras puede persistir en el corto o en el largo plazo. La dependencia de corto plazo se puede detectar cuando existe una autocorrelación significativa de los retornos en los primeros rezagos, lo cual resulta en modelos tales como, GARCH, AR y ARMA (Engle, 1982; Bollerslev, 1986). Tal como lo afirman León y Reveiz (2010), en presencia de este tipo de procesos de corto plazo, después de varias realizaciones de la variable aleatoria, estos procesos convergen en una distribución normal por el teorema del límite central.

Mandelbrot (1972) afirma que el análisis de autocorrelación es efectivo principalmente en variables que se comportan como una normal, y que son de alta frecuencia y rezagos cortos, es decir, son efectivos para modelos de corto plazo con efectos gaussianos. No obstante, los problemas gaussianos de corto plazo son importantes, no son los únicos problemas que surgen del análisis de series de tiempo. 
Por su parte, la dependencia serial de largo plazo de los retornos fue inicialmente estudiada en la complejidad de los fenómenos naturales. H.E. Hurst encontró que algunos fenómenos naturales seguían procesos con memoria de largo plazo, lo que luego se llamaría procesos aleatorios sesgados (Peters, 1992) o movimientos brownianos fractales (Mandelbrot y Hudson, 2004).

En los 70 Mandelbrot aplicó modelos que tuvieron en cuenta el fenómeno de caminata aleatoria sesgada descrito por Hurst, con los cuales, según Wilmott (2005), revolucionó la forma de pensar y la práctica de ciencias como la física estadística, meteorología y matemáticas a través de la aproximación fractal ${ }^{4}$ a la dinámica de procesos aleatorios. Mandelbrot, considerado el padre de la teoría de fractales, también estudió el comportamiento del mercado de capitales, en los cuales encontró dependencia de largo plazo en los activos financieros. Adicionalmente, autores como Peters (1992), Ambrose et ál. (1993), Cajueiro y Tabak (2008), entre otros, han corroborado los hallazgos de Mandelbrot. En particular, Peters (1992) encontró evidencia de memoria de largo plazo en los índices accionarios de Estados Unidos, y descartó que los modelos de dependencia de corto plazo (por ejemplo, GARCH y ARCH) sirvieran para capturar este fenómeno más duradero.

De acuerdo con los hallazgos de Peters (1992), una caminata aleatoria sesgada significa que hay algún grado de dependencia o "memoria" de largo plazo entre las observaciones. Esto es, que los eventos de un período influyen sobre los períodos siguientes, inclusive si ambas observaciones se encuentras distantes una de la otra. A esta dependencia de largo plazo se le conoce como persistencia cuando un movimiento positivo en el activo tiende a ser seguido por un movimiento en la misma dirección; por el contrario, cuando una serie de tiempo presenta un comportamiento anti-persistente significa que existe mayor probabilidad de que un movimiento positivo sea seguido por uno negativo. En contraste con la idea de la existencia de memoria de largo plazo, una caminata aleatoria no sesgada implica un comportamiento que puede ser descrito por un movimiento browniano en el que los eventos pasados no pueden ser utilizados para predecir el futuro, donde la probabilidad de un movimiento positivo o negativo siempre es $1 / 2$.

Al presentarse evidencia de dependencia de largo plazo o caminata aleatoria sesgada se incumple el ya mencionado supuesto de independencia sobre el que

4 Según Mandelbrot y Hudson (2004) la palabra fractal viene de fractus, que es el participio pasado del verbo partir. Un fractal es un patrón o forma cuyas partes más pequeñas replican la forma y los patrones de la estructura total. Por ejemplo, la hoja de un helecho está hecha de hojas más pequeñas, las cuales a su vez, contienen hojas aún más pequeñas.

PP. $203-245 \cdot N .^{\circ} 6 / 2011$ 
recae el MB. Muchos modelos de las finanzas modernas recaen sobre este supuesto; entre ellos el Capital Asset Pricing Model (CAPM), Arbitrage Pricing Theory (APT) y el modelo de Black and Scholes.

Por ejemplo, la hipótesis fuerte de los mercados eficientes ${ }^{55}$ (EMH por sus siglas en inglés, Efficient Market Hypothesis), que es uno de los principales pilares de las finanzas fodernas (Peters, 1989), establece que toda la nueva información es inmediata y está totalmente reflejada en los precios del mercado. Esto implica que el efecto de cualquier información nueva sobre los retornos es impredecible; la probabilidad de un movimiento positivo o negativo siempre es $1 / 2$, por lo que el mejor predictor del precio futuro es el precio actual. De esta forma, la EMH supone que los retornos se caracterizan por seguir una caminata aleatoria o un MB. Como se mencionó, en la literatura se ha mostrado que muchos activos no cumplen el supuesto de independencia y, por lo tanto, con el MB. En consecuencia, la presencia de dependencia de largo plazo invalida la EMH y la información nueva no es inmediatamente reflejada en el precio de los activos.

De acuerdo con Peters (1989) la presencia de dependencia de largo plazo en los activos se puede explicar en palabras comunes como el "sentimiento del mercado", que puede hacer que una tendencia persista en el mercado aun cuando no hay un evento en desarrollo o nuevas noticias que incorporar. Adicionalmente, León y Vivas (2010) explican que la persistencia puede asociarse al comportamiento irracional de los seres humanos, como por ejemplo cuando las decisiones de los inversionistas no son independientes y están caracterizadas por un comportamiento no lineal e imitativo, así como cuando los inversionistas no reaccionan a la nueva información de una manera continua, sino de una forma discreta y acumulada.

Greene y Fielitz (1979 y 1980) muestran que ante la presencia de dependencia de largo plazo las decisiones de inversión, de acuerdo con la relación de riesgoretorno de los activos y la composición de portafolios eficientes pueden cambiar con la elección de la frecuencia de los retornos. Incluso estos autores afirman que el CAPM solo es válido si todos los inversionistas tienen horizontes de inversión idénticos. Sobre este modelo y las implicaciones de la dependencia de largo plazo se profundizará más adelante.

5 La hipótesis fuerte de los mercados eficientes establece que, además de incorporar total e inmediatamente la información, los precios contienen información que puede ser privilegiada (Cuthbertson y Nitzsche, 2004). De acuerdo con Merkens (2007) la persistencia en los retornos de los activos puede estar reflejando la utilización de información privilegiada.

PP. 203-245 • N. ${ }^{\circ} 6 / 2011$ 
En esta sección se explicó el concepto de la caminata aleatoria, que es el supuesto que tienen en común varios modelos financieros. Adicionalmente, se documentó que algunos activos financieros presentan fenómenos de dependencia de largo plazo, lo cual invalida el supuesto de independencia sobre el que se sustenta la caminata aleatoria. A continuación se profundizará sobre el fenómeno de dependencia de largo plazo y las metodologías para medirlo.

\section{Análisis del rango reescalado y exponente de Hurst para medir la dependencia de largo plazo}

Los estudios sobre el concepto de la memoria de largo plazo comenzaron a desarrollarse a partir del análisis de algunos fenómenos naturales. En la hidrología, H.E. Hurst encontró evidencia de memoria de largo plazo en los flujos del río Nilo. Posteriormente Mandelbrot (1962) desarrolló el análisis del rango reescalado, obtuvo una aproximación al exponente de Hurst y lo aplicó a series de tiempo en campos como la meteorología, lingüística y finanzas, entre otros.

El análisis del rango reescalado se originó en la geofísica como resultado de los estudios de Hurst, quien debía encontrar la capacidad óptima de una represa en el río Nilo, la cual dependía del flujo de agua del río . Peters (1989) y Lo (1991) explican que, dada una cantidad de descargas, las dimensiones óptimas de la represa variarán con el flujo que recibe cada año. Hurst encontró que el flujo del río presentaba un grado de persistencia, el cual midió utilizando el rango reescalado.

Además de esta metodología, León y Vivas (2010) mencionan la existencia otras metodologías para medir la dependencia de largo plazo, por ejemplo: Detrended fluctuation Analysis, Wavelets y regresión basada en periodogramas ${ }^{7}$. Sin embargo, los autores escogen la metodología del rango reescalado por su "parsimonia... robustez y utilidad para detectar la dependencia de largo plazo, así como por la conveniencia de tener un acervo de literatura". Adicionalmente, el rango reescalado y el exponente de Hurst pueden ser calculados en cualquier programa que realice regresiones lineales; esto hace su implementación sencilla y puede incrementar su difusión dentro de las prácticas del mercado de capitales.

La metodología del rango reescalado de Hurst consiste en medir el rango $(R)$ de la desviación acumulada de una serie de tiempo $x_{j}$ frente a su media $\bar{x}_{j}$ durante 
un período $n$; es decir, mide cómo se comporta, a través del tiempo, el rango de la serie una vez se excluye el efecto de tendencia.

$$
R_{n}=\max _{1 \leq k \leq n}\left(\sum_{j=1}^{k}\left(x_{j}-\bar{x}_{n}\right)\right)-\min _{1 \leq k \leq n}\left(\sum_{j}^{k}\left(x_{j}-\bar{x}_{n}\right)\right)
$$

El rango depende del tiempo, de tal forma que $R$ aumenta al aumentar $n$. Con el fin de estandarizar la medida, Hurst dividió $R$ por la desviación estándar de las observaciones $(S)$. Dentro de sus hallazgos, Hurst encontró que $R / S$ podía ser estimado por la siguiente ley, que se conoce como la Ley Empírica de Hurst:

$$
\frac{R}{S_{t}}=c n^{H}
$$

Donde $H$ es el exponente de Hurst, $c$ es una constante y $n$ es el período de tiempo. Al aplicar logaritmos, con una estimación mediante mínimos cuadrados ordinarios se obtiene la estimación del exponente de Hurst $(H)$ :

$$
\log \frac{R}{S}_{n}=\log (c)+H \log (n)
$$

Mandelbrot (1965) encontró que $H$ satisfacía $0<\mathrm{H}<1$. Cuando el exponente de Hurst está entre 0.5 y 1 significa que el proceso presenta persistencia; es decir, que un retorno de signo positivo tiene una alta probabilidad de ser seguido por otro del mismo signo, y viceversa. Por su parte, un exponente de Hurst entre 0 y 0.5 implica un comportamiento anti- persistente, que algunos autores relacionan con procesos de reversión a la media, en el que un retorno positivo (negativo) tiene una alta probabilidad de ser seguido por un retorno negativo (positivo). Finalmente, si el exponente es igual a 0.5 , el proceso sigue una caminata aleatoria y los retornos de hoy no tienen influencia sobre los retornos futuros. No obstante, el exponente de Hurst de una serie independiente se aproxima asintóticamente a 0.5 , al utilizar series finitas el exponente puede resultar mayor a 0.5 .

Dada su naturaleza asintótica, cuando la estimación del exponente de Hurst se realiza sobre series finitas se origina un sesgo que sobreestima el exponente (León y Vivas, 2010; León y Reveiz, 2010 y Peters, 1994). Varios autores (Peters, 1994; Ellis; 2007; Couillard y Davison, 2005) han realizado ajustes al exponente de Hurst PP. 203-245 - N. ${ }^{\circ} 6 / 2011$ 
y a la metodología del rango reescalado. De acuerdo con León y Vivas (2010), con el ajuste propuesto por Anys y Lloyd (1976) y Peters (1994) para tener en cuenta el sesgo por el uso de muestras finitas se evita sobrestimar la magnitud del alejamiento respecto de la hipótesis nula de independencia como consecuencia de este sesgo.

El exponente ajustado se calcula con la siguiente ecuación:

$$
H_{a d j}=H-\left(H_{f}-0,5\right)
$$

Donde $H_{f}^{10}$ se obtiene a partir del valor esperado del rango reescalado $E \frac{R}{S}{ }_{n}$ para una ventana de datos independiente de tamaño $(n)$, de la cual resulta el exponente de Hurst de una serie independiente de tamaño N. Este valor esperado presenta un sesgo positivo que crece de manera inversa con el tamaño de dicha ventana, según lo proponen Anys y Lloyd (1976):

$$
E \frac{R}{S_{t}}=\frac{n-\frac{1}{2}}{n \sqrt{n \pi / 2}} \sum_{i=1}^{n-1} \sqrt{\frac{n-1}{i}}
$$

Como lo mencionan León y Vivas (2010), este ajuste permite estimar el sesgo por el uso de series de tiempo finitas en el rango reescalado, y de esta forma, permite utilizar las estimaciones del exponente de Hurst con el fin de evaluar cuantitativamente el impacto de la dependencia de largo plazo en los principales modelos de valoración de activos y optimización de portafolio. Este documento utilizará la estimación del exponente de Hurst ajustado (sin sesgo por uso de muestras finitas), para contrastar los resultados del modelo CAPM clásico, con los que incorporan el modelo el efecto de dependencia de largo plazo.

Sin embargo, esta metodología supone que el exponente de Hurst es estable en el tiempo. León y Reveiz (2011), citando a Di Mateo (2007), afirman que este exponente puede variar en el tiempo, lo que significaría que la estructura que siguen los activos no es uni-fractal sino multi-fractal. A pesar de lo anterior, la estimación de un exponente de Hurst confiable debe hacerse sobre una serie larga de datos, por lo que estimar exponentes que varíen a través del tiempo requiere series de longitud considerablemente largas.

Con base en esta metodología, se realizó la prueba gráfica de Mandelbrot para tres acciones del mercado colombiano y para el Índice General de la Bolsa de 
Colombia (IGBC). Esta prueba consiste en mostrar el comportamiento del rango reescalado con la ventana de tiempo, cuya relación lineal es el exponente de Hurst estimado. Adicionalmente, se presenta la estimación del exponente de Hurst ajustado con la metodología de Anys y Loyd (1976) y Peters (1994).

Para el mercado colombiano se escogieron las acciones de Nutresa (antes Nacional de Chocolates), Éxito y Bancolombia; el criterio para su elección se basó en la longitud de la serie y en la liquidez de la acción, además de ser acciones representativas de diferentes sectores económicos. Los datos de los precios de acciones y del IGBC se obtuvieron de Bloomberg, desde el 9 de marzo de 1998 hasta el 9 de mayo de $2011^{8}$, para un total de 3436 observaciones diarias 9 .

Gráfico 1 (Acción Nutresa)

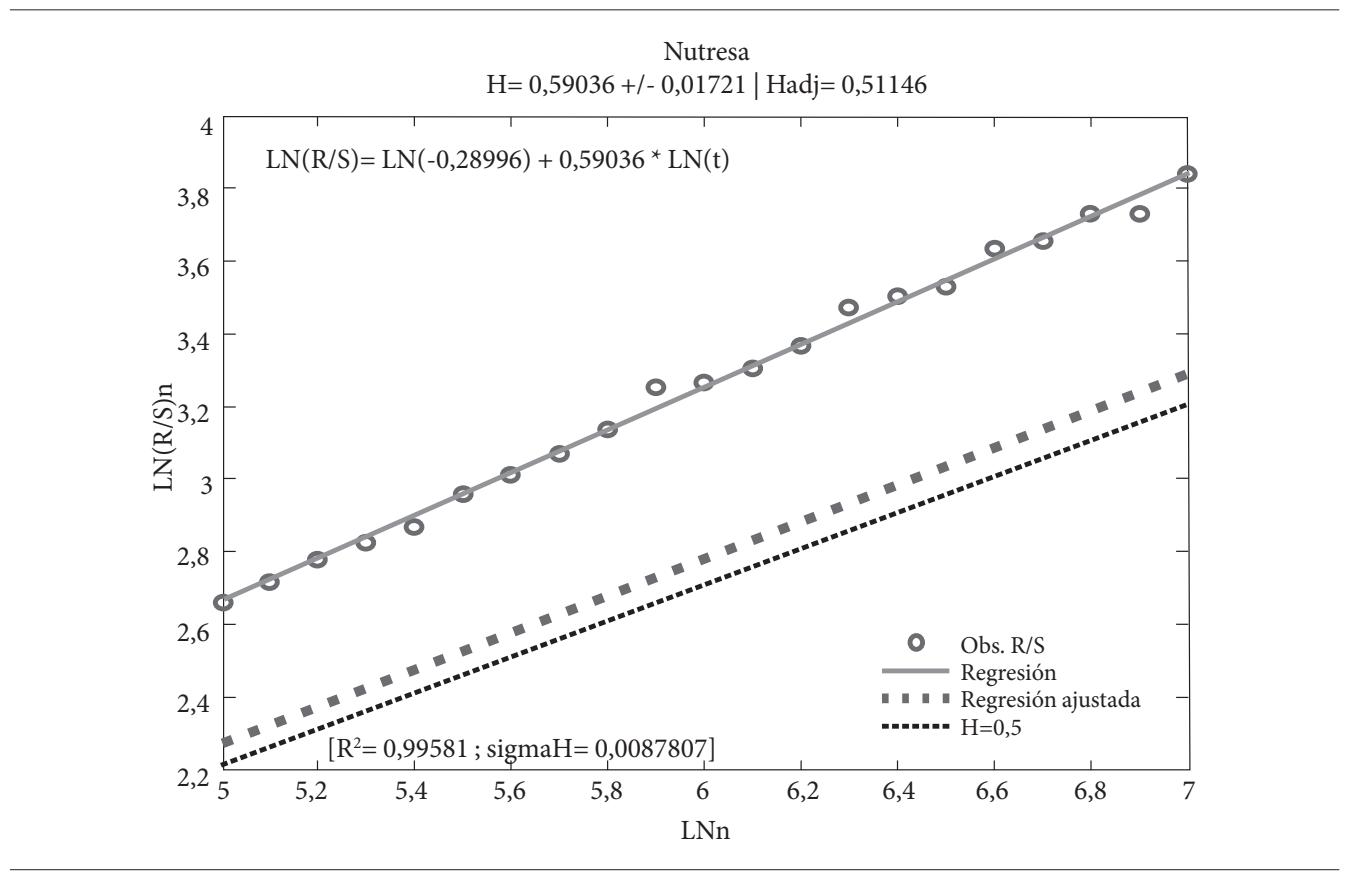

8 Debido a que la Bolsa de Valores de Colombia (BVC) nació en 2001, producto de la fusión de las bolsas de Bogotá, Medellín y Occidente, los datos del IGBC antes del 3 de julio del 2001 corresponden a un empalme de las series de los índices de las bolsas de valores antes mencionadas.

9 La longitud de las series analizadas no es lo suficientemente extensa y por esa razón no se estima más de un exponente y se supone que este es estable en el tiempo. 


\section{Gráfico 2 (Acción Éxito)}

Éxito

$\mathrm{H}=0,59192+/-0,02344 \mid \mathrm{Hadj}=0,51302$

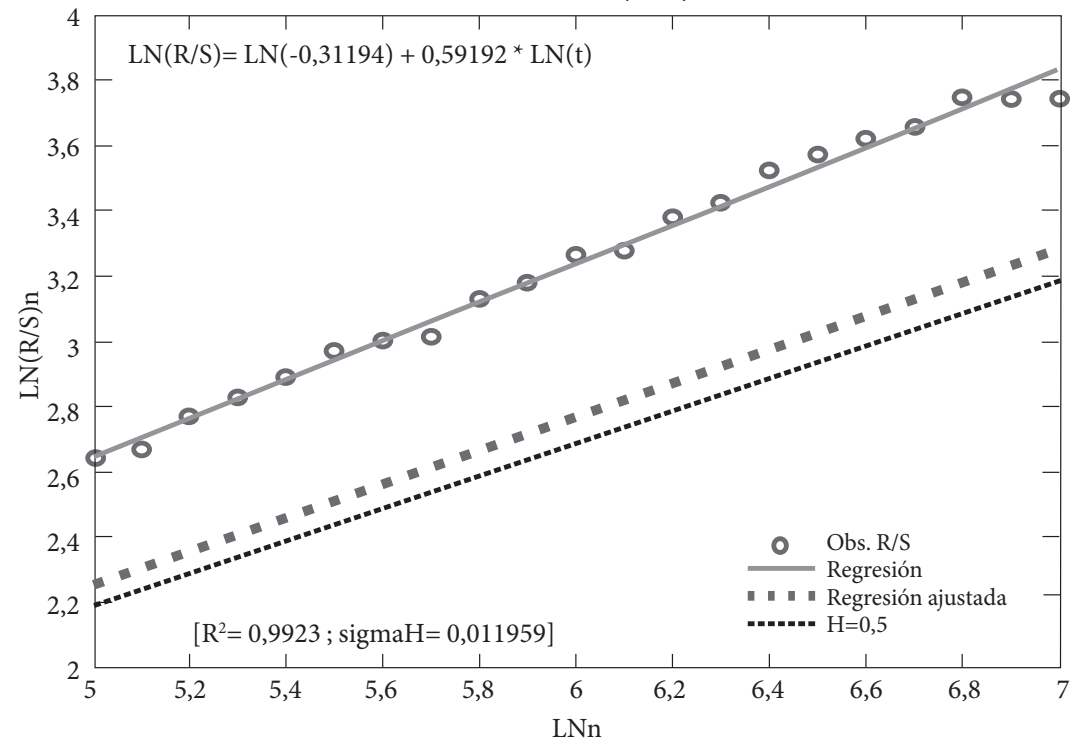

Gráfico 3 (Acción Bancolombia)

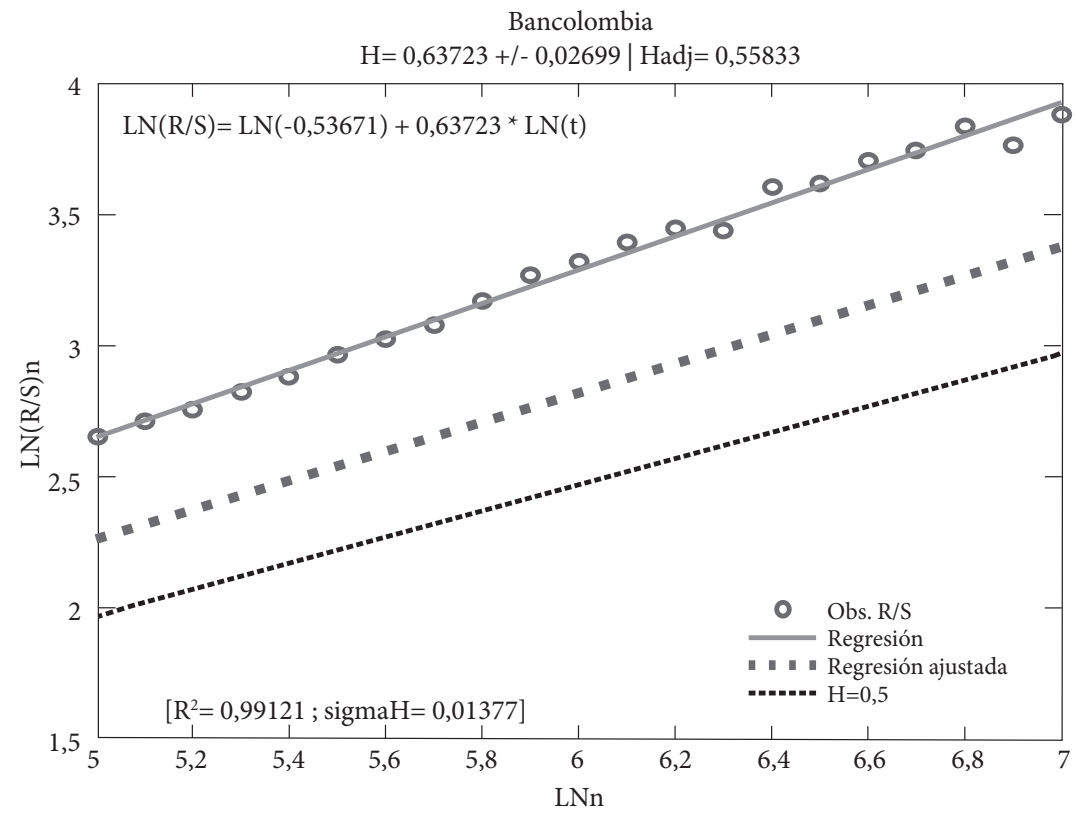




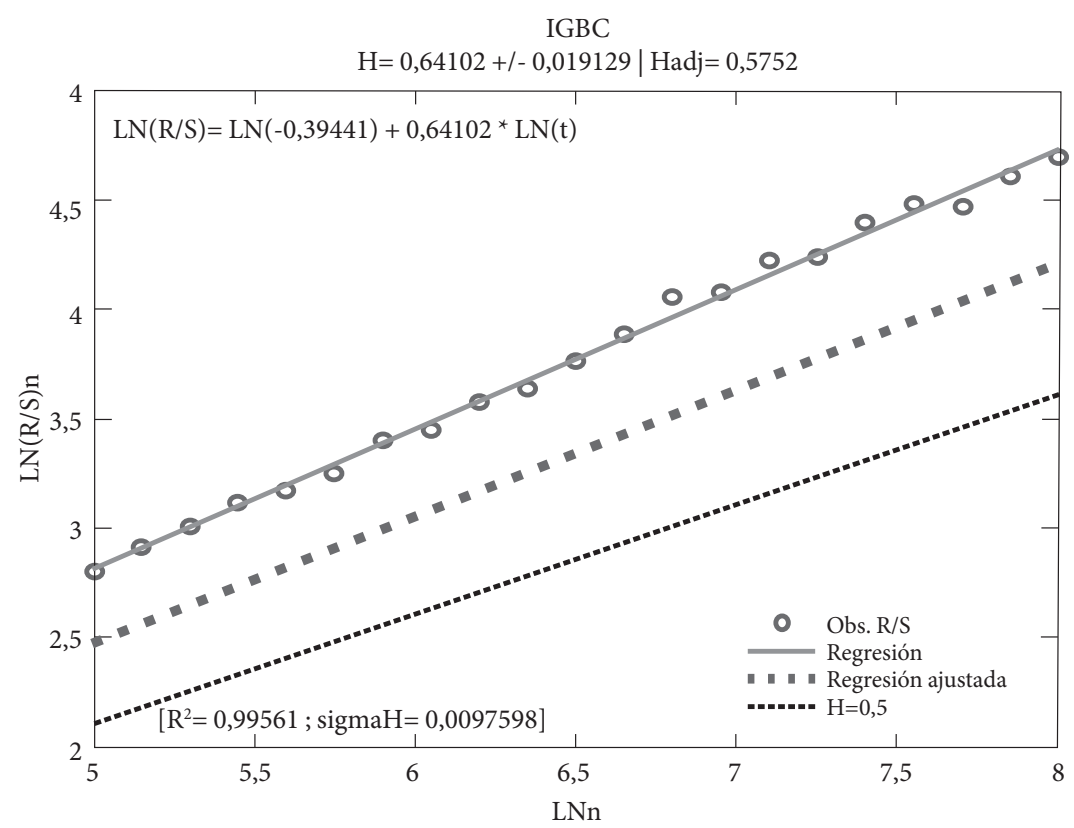

Fuente: Cálculos del autor

Las tres acciones analizadas y el IGBC muestran persistencia ${ }^{10}$, lo que se refleja en que las estimaciones de los exponentes de Hurst arrojan resultados por encima de 0.5 (Nutresa: 0.59, Éxito: 0.59, Bancolombia: 0.63 e IGBC: 0.64). Esto implica que un avance (retroceso) en el precio de estas acciones probablemente será seguido por otro movimiento de signo positivo (negativo) incluso al alejarse una observación de otra. Al realizar el ajuste por el sesgo de estimación se puede observar que el grado de persistencia de las acciones de Nutresa (exponente de Hurst ajustado: 0.51) y Éxito (exponente de Hurst ajustado: 0.51) disminuye, sin embargo, continúa estando por encima de 0.5. Asimismo, los exponentes ajustados de Bancolombia y del IGBC continúan mostrando persistencia, superior a la persistencia de Nutresa y Éxito.

Adicionalmente, se realizaron pruebas de significancia a los exponentes de Hurst estimados. Las pruebas de significancia desarrolladas por Peters (1994), Ellis (2007), Couillard y Davison (2005) pueden ser aplicadas sobre el exponente

10 León y Vivas (2010) también encuentran persistencia para este índice. 
de Hurst debido a que estos autores encontraron que se distribuye como una normal. La prueba de significancia utilizada, también explicada en el documento de León y Vivas (2010), muestra que la acción de Bancolombia, el IGBC, la acción de Walmart y el S\&P500 rechazan la hipótesis nula de independencia, que en otras palabras significa que el grado de alejamiento del supuesto de independencia es estadísticamente relevante (Ver Anexo 1).

Cuadro 1. Exponente de Hurst ajustado y prueba de significancia

\begin{tabular}{|l|l|c|c|}
\hline \multicolumn{1}{|c|}{ Activo } & & Hadj & Estadístico t \\
\hline \multirow{4}{*}{ Colombia } & Nutresa & 0,511 & 0,419 \\
\cline { 2 - 4 } & Éxito & 0,513 & 0,463 \\
\cline { 2 - 4 } & Bancolombia & 0,558 & $2.206^{*}$ \\
\cline { 2 - 4 } & IGBC & 0,575 & $3.012 * * *$ \\
\hline \multirow{5}{*}{ Estados Unidos } & JPMorgan & 0,486 & $-0,537$ \\
\cline { 2 - 4 } & Walmart & 0,454 & $-1.859^{*}$ \\
\cline { 2 - 4 } & Kellogs & 0,467 & -1.324 \\
\cline { 2 - 4 } & S\&P500 & 0,451 & $-1.968^{* *}$ \\
\hline
\end{tabular}

* Significado al 90\% de confianza, ** (95\%), *** (99\%).

Fuente: cálculos del autor.

\section{Capital Asset Pricing Model (CAPM)}

En las secciones anteriores se mostró que muchos de los principales modelos de la teoría financiera recaen sobre el supuesto del MB, el cual carece de validez según los hallazgos de la dependencia de largo plazo en algunas series de tiempo de activos financieros. Posteriormente se expuso la metodología del rango reescalado como medida para cuantificar la memoria de largo plazo. En esta sección se quiere mostrar el Capital Asset Pricing Model (CAPM), sus supuestos, cuál es el impacto de la dependencia de largo plazo en el desarrollo del modelo, y cómo debe cambiar su formulación para tener en cuenta este efecto.

El CAPM es uno de los pilares de la teoría financiera moderna y fue el primero en introducir la noción de riesgo dentro de un modelo de equilibrio general para la valoración de activos financieros (Litterman, 2003). Este modelo, desarrollado por Treynor, Sharpe, Lintner y Mossin en los años 60, es un estándar para la valoración de activos del que se extrae el retorno esperado de una acción y así, a 
partir de esa medida, se calcula el costo de capital o se evalúa el desempeño de un portafolio (Campbell et ál., 1997). Adicionalmente, el CAPM provee un valor de referencia para evaluar si el precio de una acción se encuentra por debajo o por encima de su valor de equilibrio.

Este modelo es un estándar en los programas de enseñanza de finanzas corporativas. No obstante, además de ser enseñado en la academia, es ampliamente utilizado en la práctica. Mandelbrot y Hudson (2004) mencionan que una encuesta realizada por dos estudiantes de la Universidad de Duke a 392 gerentes financieros de compañías pertenecientes al U.S Fortune 500, mostraba que en 1999 el $73.5 \%$ de los preguntados estimaban el costo del capital con el CAPM. De forma similar, en Europa, en 2001, el porcentaje ascendió a 77\%.

El CAPM está basado en la teoría de equilibrio económico, que busca cuál debe ser el exceso de retorno esperado de un activo frente a la tasa libre de riesgo, de tal forma que la demanda y la oferta de ese activo se igualen. La oferta en el modelo es simplificada como la capitalización de mercado estimada ${ }^{1111}$. A su vez, la demanda proviene de la maximización de la utilidad de un agente averso al riesgo que escoge el porcentaje de su riqueza para invertir en diferentes activos. En equilibrio, el portafolio óptimo de mercado es aquel que corresponde a la capitalización (oferta) y consigue vaciar el mercado.

El modelo supone que los agentes son aversos al riesgo y maximizan su utilidad esperada (en el sentido de von Neumann-Morgenstern) a través de decisiones de inversión basadas en el criterio de maximización del retorno esperado y minimización de la varianza (Markowitz , 1959). Además, en el modelo se supone que todos los mercados son eficientes y perfectos (no hay oportunidades de arbitraje), no hay costos de transacción y todos los inversionistas tienen la misma información. Por otra parte, las tasas de depósitos y préstamos se suponen iguales, y se asume que en el mercado monetario todos los agentes tienen capacidad ilimitada de prestar y pedir prestado a la tasa libre de riesgo. Adicionalmente, las expectativas son homogéneas en el mercado; es decir, todos los agentes tienen las mismas oportunidades de inversión y ven los retornos esperados y las desviaciones estándar de los activos de la misma forma.

Sumado a lo anterior, el modelo supone que todos los inversionistas tienen un mismo horizonte de inversión, donde este supuesto recae en la existencia de neutralidad en el horizonte de inversión. Es decir, en el sentido de Campbell et

11 Número de acciones o cantidad circulante del activo multiplicada por el precio de mercado. PP. $203-245 \cdot N .{ }^{\circ} 6 / 2011$ 
ál. (1997), es necesario suponer que los activos siguen un proceso de caminata aleatoria, en el que los retornos son independientes e idénticamente distribuidos y conjuntamente se distribuyen como una normal multivariada.

Sean $N$ activos riesgosos $j=1,2 \ldots N$, dadas unas dotaciones iniciales de rique$\mathrm{za}$, los retornos de los activos (П) y la matriz de varianzas y covarianzas entre los activos $(\Sigma)$, los agentes escogen la distribución de su dotación $(\omega)$ maximizando la función de utilidad que, como se muestra en la ecuación (8), depende positivamente del retorno del portafolio y negativamente de la varianza de este ${ }^{12}$. Adicionalmente, el coeficiente $\lambda$, que multiplica a la varianza, determina la des-utilidad que le produce al agente la volatilidad de los activos; en otras palabras, representa el coeficiente de aversión al riesgo.

$$
\begin{gathered}
\max _{(\omega)} U=E\left[\prod \omega\right]-0,5 \lambda\left(\omega \Sigma \omega^{\prime}\right) \\
\text { s.a. } \sum_{j-1}^{N} \omega_{j}=1
\end{gathered}
$$

De la condición de primer orden se obtiene:

$$
\omega=\frac{1}{\lambda}\left(\Sigma^{-1} \Pi\right)
$$

La intuición detrás de la ecuación (9) es que la proporción de la riqueza invertida en el activo riesgoso depende negativamente del coeficiente de aversión al riesgo, tal que a mayor aversión al riesgo la cantidad invertida en el activo riesgoso es menor. De igual forma, la inversión en el activo riesgoso depende negativamente del riesgo de los activos representado por la matriz de varianza-covarianza. Finalmente, la decisión de invertir en los activos riesgosos depende positivamente del retorno de estos, es decir, a mayor retorno de los activos riesgosos mayor es la inversión en ellos.

12 Se supone la función de utilidad cuadrática porque representa la disyuntiva a la que se enfrenta el inversionista entre retorno y riesgo. Litterman (2003) afirma que una función cuadrática puede ser una aproximación de una función de utilidad general suavizada. Adicionalmente, esta función permite representar el comportamiento de un inversionista con una aversión al riesgo relativa constante y para el cual los retornos se distribuyen como una normal. 
Como en el modelo se suponen expectativas homogéneas e información simétrica, los inversionistas tienen los mismos retornos esperados, y la única diferencia en la optimización del portafolio será la aversión al riesgo. A partir de este coeficiente, los inversionistas decidirán la cantidad invertida en activos riesgosos (acciones) en relación con activos libres de riesgo (bonos).

A partir de la optimización de los inversionistas, la ecuación principal del CAPM establece que el retorno esperado del activo $j$ durante un horizonte de inversión ${ }^{13}$ está dado por la ecuación (10).

$$
E\left(R_{j}\right)=R_{f}+\beta_{j} E\left(R_{m}\right)-R_{f}
$$

Esta relación implica que el retorno esperado de equilibrio de un activo riesgoso (j) es una función lineal y positiva de su covarianza $\beta_{j}=\frac{\sigma_{j, m}}{\sigma_{m}^{2}}$ con la prima de riesgo que es igual al rendimiento esperado del portafolio de mercado $(m)$ menos la tasa libre de riesgo, donde $\sigma_{j, m}$ es la covarianza entre el activo $j$ y el mercado, y $\sigma_{m}^{2}$ es la varianza del mercado.

Reescribiendo la ecuación anterior, en términos de excesos de retornos sobre la tasa libre de riesgo, se tiene que el exceso de retorno del activo $j$ es $\beta_{j}$ veces proporcional a la prima de riesgo del mercado:

$$
{ }_{j}=\beta_{j \quad m}
$$

Donde $\mu_{j}$ es el exceso de retorno del activo $j, \mu_{m}$ es el exceso de retorno del portafolio de mercado y $\beta_{j}$, definida de la misma forma que en la ecuación (10), es una forma de medir el riesgo de un activo individual como una combinación lineal con el riesgo del portafolio de mercado. Según Greene y Fieltz (1980), $\beta_{j}$ es un indicativo del riesgo sistemático ${ }^{14}$ de un activo individual.

13 Debido a que se supone neutralidad en el horizonte de tiempo la escogencia del período de inversión no es relevante; el horizonte de inversión es especificado en el modelo, pero corresponde al período de estimación del retorno del activo riesgoso y el libre de riesgo.

14 El riesgo sistemático es el riesgo asociado a los movimientos del mercado, y se mide como la covarianza del portafolio con el portafolio de mercado. En contraposición, el riesgo no sistemático está asociado solo a un activo o a un grupo pequeño de activos (Ross, 2005). 
La idea subyacente al horizonte de inversión único ${ }^{15}$ en el que se desarrolla el CAPM está basada en el supuesto de neutralidad del horizonte de tiempo, según el cual los activos siguen un MB. Esto implica que, ante la independencia de los retornos en el tiempo, la consistencia temporal de la volatilidad se mantiene y se puede aplicar la regla de la raíz de tiempo y el horizonte de inversión es irrelevante. No obstante, en la realidad la frecuencia de los datos y el horizonte de inversión elegido tienen relevancia.

Debido a que la evidencia ha mostrado que los retornos de los activos no son independientes en el tiempo, las medidas de riesgo de los activos pueden cambiar al incorporar la dependencia de largo plazo en los retornos. En particular, Greene y Fieltz (1979 y 1980) muestran cómo la existencia de un movimiento browniano fractal puede cambiar la formulación del CAPM.

Sea $B(t)$ un movimiento browniano con incrementos $B(t+5)-B(t)$, siendo $s$ el tamaño del incremento en el tiempo (horizonte de inversión), estos son idénticamente distribuidos $(\triangleq)$ a la raíz cuadrada del intervalo de tiempo $s$ multiplicado por un incremento de una unidad (Greene y Fieltz, 1980).

$$
B(t+s)-B(t) \triangleq s^{0,5} B(t+1)-B(t) \quad \forall t, s
$$

Esto es equivalente a lo que se mostró en la sección anterior respecto a la regla de la raíz cuadrada del tiempo (1). En el caso de un movimiento browniano fractal $B_{H}$, Greene y Fieltz (1980) muestran que la ecuación (12) se convierte en (13).

$$
B_{H}(t+s)-B_{H}(t) \triangleq s^{H} \quad B_{H}(t+1)-B_{H}(t) \quad \forall t, s
$$

Donde $H$ es el exponente de Hurst expuesto en la sección III. De esta forma, si se compara la ecuación (13) con la (12), se tiene que un movimiento browniano fractal es un movimiento browniano ordinario (o una caminata aleatoria) cuando $H=0.5$; es decir, el movimiento browniano fractal es una generalización del movimiento browniano (León y Reveiz, 2011).

A partir de las ecuaciones anteriores, Greene y Fieltz (1979 y 1980) muestran que la varianza y la covarianza de un movimiento browniano fractal están dadas por

15 Nótese que la formulación del CAPM clásico no contiene subíndices que indican el tiempo. Por lo que el horizonte de inversión al que se aplique el modelo es irrelevante en la teoría. 
(14) y (15), donde la varianza y covarianza del movimiento browniano fractal (14) es una generalización de la varianza y covarianza del movimiento browniano (2).

$$
\begin{aligned}
& \operatorname{Var} B_{H}(t+s)-B_{H}(t)=s^{2 H} \operatorname{Var} B_{H}(t+1)-B_{H}(t) \\
& \operatorname{Cov}\left\{B_{H_{j}}(t+s)-B_{H_{j}}(t), B_{H_{o}}(t+s)-B_{o}(t)\right\} \\
= & s^{H_{j}+H_{o}} \operatorname{Cov}\left\{B_{H_{j}}(t+1)-B_{H_{j}}(t), B_{H_{o}}(t+1)-B_{H_{o}}(t)\right\}
\end{aligned}
$$

Donde $H_{j}$ y $H_{o}$ son los exponentes de estimados Hurst para cada una de las series. La ecuación (15) implica que la covarianza entre los retornos de dos activos $\left(j \mathrm{y} o\right.$ ) en el horizonte de inversión $s$ es $s^{H_{j}+H_{o}}$ veces la covarianza entre los retornos de esos activos en un intervalo de una unidad.

Debido al supuesto de neutralidad del horizonte de inversión en el CAPM, un inversionista con un horizonte de inversión $s$ que quiere calcular cuánto es el retorno esperado de una acción durante ese período y que supone que los activos son independientes en el tiempo, utilizaría la ecuación (10), diseñada para la inversión en un solo período, de la forma:

$$
\begin{gathered}
E\left(R_{f, s}\right)=R_{f, s}+\beta_{j, s} E\left(R_{m, s}\right)-R_{f, s} \\
\beta_{j, s}=\frac{s^{0,5+0,5} \sigma_{j, m}}{s^{2 \times 0,5} \sigma_{m}^{2}}=\frac{s \sigma_{j, m}}{s \sigma_{m}^{2}}
\end{gathered}
$$

Donde $E\left(R_{r, s}\right)$ es el retorno esperado del activo $j$ durante el período $s ; E\left(R_{m, s}\right)$ es el retorno esperado para el portafolio del mercado durante el mismo período; $R_{f, s}$ es un retorno libre de riesgo; y $\sigma_{j, m}$ y $\sigma_{m}^{2}$ son la covarianza entre los retornos del activo $j$ y el mercado, y la varianza de los retornos del mercado, respectivamente.

En la práctica estos cálculos se realizan basados en retornos diarios que son escalados al período de inversión $s$. Cuando la frecuencia de los datos difiere del período de inversión y las series financieras presentan dependencia de largo plazo (es decir, no se cumple el supuesto de neutralidad del horizonte de inversión), la ecuación (16) cambia para tener en cuenta ese efecto. Basándose en las ecuaciones (14) y (15), Greene y Fieltz (1980) muestran que en presencia de la dependencia de largo plazo la ecuación (16a y 16b) se generaliza de la siguiente manera: 


$$
\begin{gathered}
E\left(R_{f, s}\right)=R_{f, s}+\beta_{j, s} E\left(R_{m, s}\right)-R_{f, s} \\
\beta_{j, s}=\frac{s^{H_{m}+H_{j}} \sigma_{j, m}}{s^{2 H_{m}} \sigma_{m}^{2}}
\end{gathered}
$$

El impacto de la dependencia de largo plazo en el modelo está en el coeficiente $\beta_{j}$, que es el ponderador del riesgo del activo frente al riesgo del portafolio. Cuando los retornos son independientes, es decir que $H_{m}=H_{j}=0,5$, el $\beta_{j}$ no cambia cuando se utilizan datos cuya frecuencia difiere del período de inversión. Otro caso en el que $\beta_{j}$ no cambia y el modelo es neutral a la elección del horizonte de inversión es cuando $H_{m}=H_{j}$; en este caso la ecuación (18) muestra que los exponentes de la ecuación (17b) se cancelan:

$$
\beta_{j, s}=\frac{s^{H_{m}} s^{H_{m}} \sigma_{j, m}}{s^{H_{m}} s^{H_{m}} \sigma_{m}^{2}}=\frac{\sigma_{j, m}}{\sigma_{m}^{2}}
$$

Por lo general, en los casos en los que existe dependencia de largo plazo, que según la evidencia presentada es un hecho común en las series financieras, se tiene que $H_{m} \neq H_{j} \neq 0,5$. Lo anterior implica que $\beta_{j}$, que es el parámetro estimado del riesgo sistemático mediante mínimos cuadrados ordinarios, cambia cuando hay dependencia de largo plazo. Esto, según Greene y Fieltz (1980), implica que los estimadores del riesgo sistemático $\beta_{j}$ deben estar medidos sobre un incremento de tiempo $s$ exactamente igual al horizonte de inversión.

Por lo anterior, al tener en cuenta la dependencia de largo plazo en la formulación del CAPM, el retorno esperado del activo $j$ cambia frente a la formulación del CAPM clásico que supone neutralidad en el horizonte de inversión e independencia serial de los retornos. A continuación se estimará el efecto cuantitativo de la existencia de la dependencia de largo plazo sobre los retornos esperados de algunas acciones del mercado colombiano y de los Estados Unidos usando el CAPM.

\section{Resultados}

Como se mencionó en las secciones anteriores, el CAPM se utiliza en la práctica para hallar el costo del capital, que es un insumo indispensable en la valoración de empresas, entre otras aplicaciones. De ahí la importancia de ajustar el CAPM con la 
formulación de la sección anterior con el fin de incorporar la dependencia serial de largo plazo de los activos. En esta sección se compararán los resultados entre los retornos esperados utilizando el CAPM clásico con los retornos esperados del modelo ajustado según las ecuaciones (17a y 17b), donde el exponente de Hurst corresponde con la versión ajustada de León y Vivas (2010) y León y Reveiz (2010). Como se explicó, la versión ajustada del exponente de Hurst corrige el sesgo derivado de la estimación del exponente sobre series finitas, lo que permite su uso práctico.

Para realizar este ejercicio se tomaron las acciones del mercado colombiano presentadas en la sección II (Nutresa, Bancolombia y Éxito). Adicionalmente, con el propósito de realizar comparaciones, se utilizarán tres acciones que cotizan en el New York Stock Exchange (NYSE) y se encuentran listadas en el índice bursátil de las 500 empresas más importantes de los Estados Unidos, el S\&P500: JPMorgan (JPM), Kellog's (K) y Walmart (WMT). Los índices que representan el portafolio de mercado son el IGBC y el S\&P500, para Colombia y Estados Unidos, respectivamente.

Al estimar los exponentes de Hurst ajustados para los activos mencionados se encuentra evidencia de dependencia de largo plazo (ver Cuadro 1), aunque solo Bancolombia, el IGBC y el S\&P500 presentan un grado de alejamiento significativo con respecto de la hipótesis nula de independencia. No obstante, se estimarán los retornos esperados para todas las acciones debido a que los resultados significativos de dependencia de largo plazo para los índices accionarios afectan la medición del riesgo sistémico de cada una de las acciones.

Para el componente libre de riesgo del modelo, la tasa libre de riesgo de EE.UU. se calculó como el rendimiento diario del índice de notas y bonos del Tesoro Americano de 0 a 3 meses calculado por Merrill Lynch. Para Colombia, el rendimiento libre de riesgo (en pesos colombianos) se aproximó como la tasa libre de riesgo de EE.UU. más la prima de riesgo país medida por el EMBI Colombia y la devaluación de la tasa de cambio promedio peso-dólar ${ }^{16}$. Los datos del índice de Merril Lynch, el EMBI Colombia y la tasa de cambio promedio fueron extraídos de Bloomberg.

Una primera aproximación al efecto de la dependencia de largo plazo sobre las decisiones de inversión se puede observar en la comparación retorno-riesgo ${ }^{17}$ de

16 Para Colombia no se utilizó el rendimiento de los títulos del gobierno TES debido a que estos activos no son considerados refugio en el mercado local, lo cual se evidencia en una relación directa entre el rendimiento de las acciones (activos riesgosos) y los TES.

17 De acuerdo con el criterio de media-varianza de Markowitz, un activo con un mayor retorno y menor riesgo domina a uno con un retorno similar, pero mayor riesgo.

PP. 203-245 • N. ${ }^{\circ} 6 / 2011$ 
estos activos. Ante la presencia de activos que siguen un movimiento browniano fractal, el riesgo del activo puede estar subestimándose o sobreestimándose al escalar los retornos de los activos a diferentes frecuencias. En consecuencia, León y Vivas (2010) muestran que la regla de la raíz de tiempo no es una forma correcta de escalar la volatilidad de un activo que presenta dependencia de largo plazo, y proponen que la volatilidad sea escalada utilizando el exponente de Hurst. De esta forma, el riesgo (desviación estándar) del activo $j$ en el período $s$, está dado por:

$$
\sigma_{j, s}=s^{H_{j}} \sigma\left(r_{j, d}\right)
$$

Donde $r_{j, d}$ son los retornos logarítmicos del activo $j$ en el período $d$, y $H_{j}$ es el exponente de Hurst ajustado para ese mismo activo.

En los Gráficos 5 al 7 se ilustra la relación retorno riesgo basada en retornos diarios de las acciones analizadas para tres períodos de inversión: un día, un mes (20 días) y un año (250 días). En estos Gráficos se evidencia que en presencia de dependencia de largo plazo (Ver Cuadro 1) la relación de retorno riesgo de los activos puede cambiar. Cuando el horizonte de inversión es un día, la frecuencia de los retornos es igual al horizonte de inversión (e.g. cuando $s=1$ ), por lo que la relación retorno riesgo no cambia (Gráfico 5).

Gráfico 5

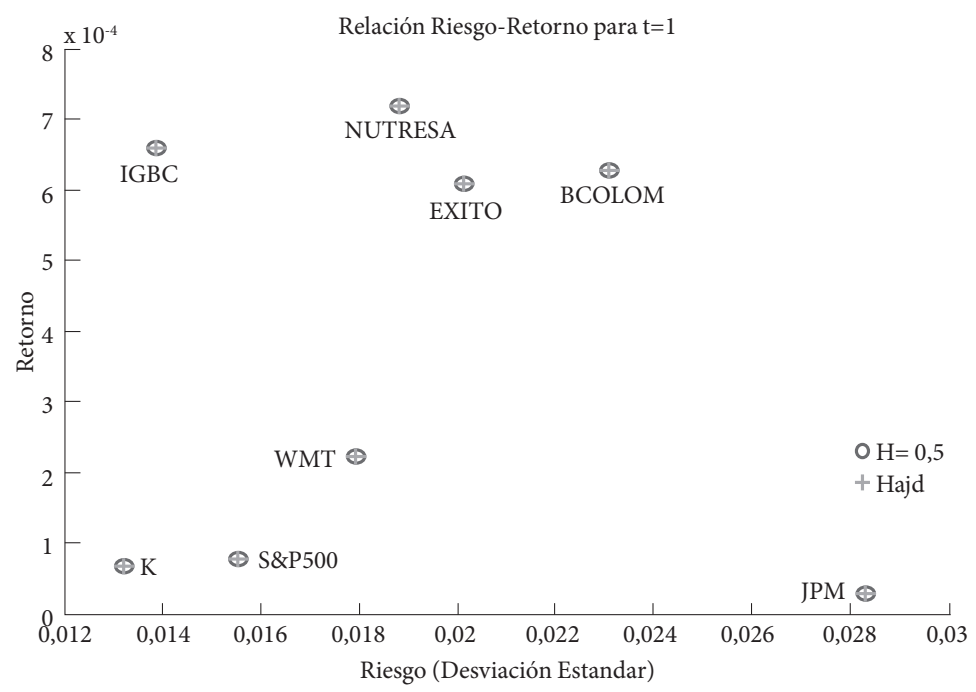


Sin embargo, para horizontes de inversión mayores (a un mes y un año), se pueden observar cambios en la relación retorno riesgo de las acciones analizadas. A partir de las pruebas de significancia, las diferencias en las relaciones retorno riesgo de Bancolombia y Walmart son estadísticamente significativas. Al ajustar el riesgo por dependencia se puede observar que al escalar la volatilidad bajo el supuesto de independencia de largo plazo, el riesgo de la acción de Bancolombia se subvalora en términos relativos y un modelo de optimización hubiera sobreestimado la asignación sobre esta acción. De otra parte, la acción de Walmart muestra el comportamiento contrario al de Bancolombia, pues al ajustar el riesgo por dependencia de largo plazo, se encuentra que el riesgo estaba sobrevalorado por lo que en un modelo de optimización su asignación se pudo haber subestimado.

Gráfico 6

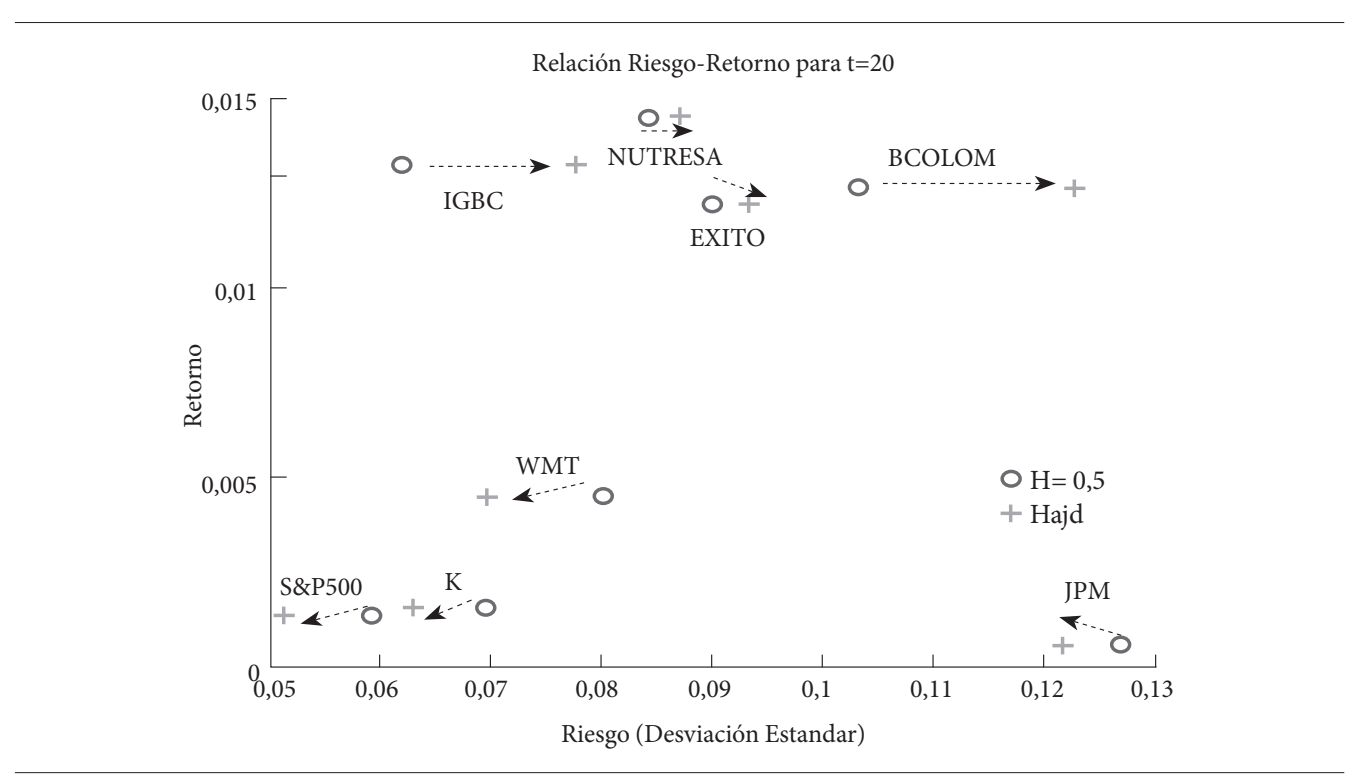

Para las acciones del mercado colombiano analizadas, no tener en cuenta la memoria de largo plazo implica una subvaloración del riesgo por la presencia de persistencia. Por el contrario, la presencia de antipersistencia resulta en que el riesgo de las acciones estadounidenses es sobreestimado. 


\section{Gráfico 7}

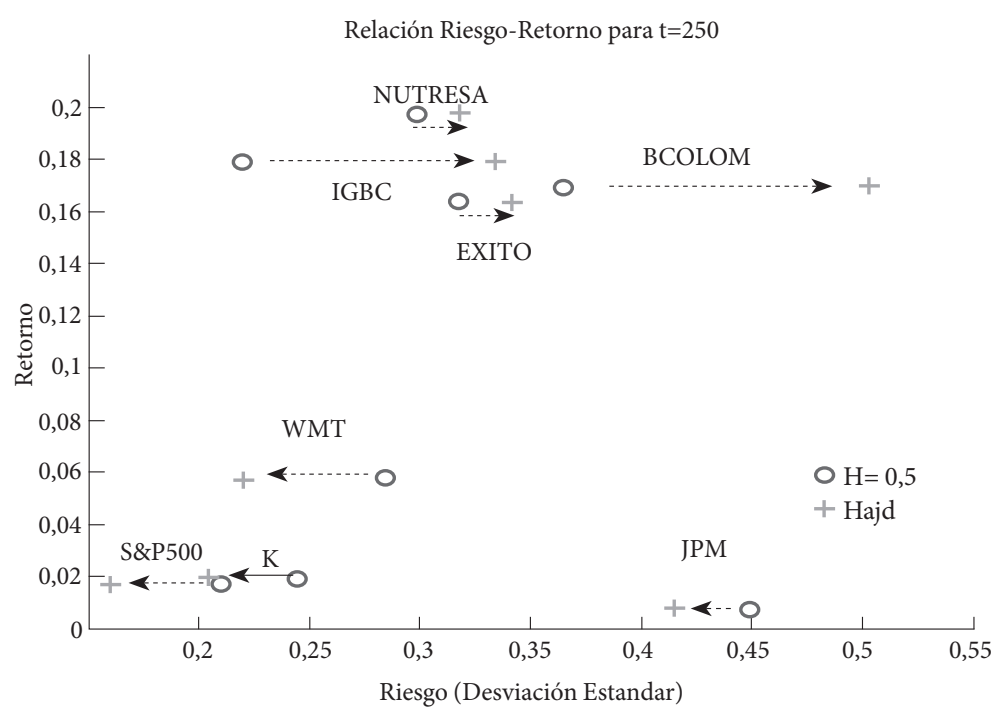

Fuente: Cálculos del autor

Además de afectar la comparación del riesgo con otros activos, la dependencia de largo plazo afecta también la relación de riesgo con el mercado. En el CAPM esto implica que al medir el retorno esperado de una acción en función de la prima de riesgo del mercado, los resultados de los retornos esperados cambian no solo cuando la acción presenta dependencia de largo plazo, sino también cuando este fenómeno se presenta en los retornos del portafolio de mercado (ecuación (17)).

Con el fin de cuantificar las diferencias entre el $\beta_{j}$ que resulta del ajuste en (17a) y (17b), frente al que no lo incorpora (16), se realizaron 5000 simulaciones a partir de ventanas de 250 datos ${ }^{18}$ para calcular las distribuciones de los betas estimados para cada uno de los plazos de inversión (un día, un mes y un año ${ }^{19}$ ) y así estimar los retornos esperados de las acciones analizadas.

Los Gráficos 8 al 10 muestran los gráficos de probabilidad de los betas ajustados por dependencia de largo plazo, frente a los betas que suponen independencia, para un período de un año. Los betas de las acciones Nutresa, Éxito y Bancolombia que

18 En el mercado usualmente se utiliza un año como plazo para aplicar este tipo de modelos. Se realizaron ejercicios con ventanas de 100 y 500 datos con resultados similares, donde las conclusiones no variaban.

19 Se reportarán los resultados para un año. 
incorporan dependencia de largo plazo difieren de los estimados sin este ajuste, y en los tres casos la línea ajustada se encuentra a la izquierda de la que no incorpora la dependencia de largo plazo, lo cual se debe a que el exponente de Hurst del IGBC es mayor que el de las acciones. Para Nutresa y Éxito la diferencia entre los betas es mayor que en la de Bancolombia debido a que la diferencia entre el exponente de Hurst de Bancolombia y del IGBC es menor.

Pese a que el exponente de Hurst de las acciones de Nutresa y Éxito no es significativamente diferente de 0.5 , la persistencia del índice accionario hace que el ajuste, por dependencia de largo plazo, muestre que el beta de estas acciones se estaba sobrevalorando, ya que el riesgo sistemático de la acción era inferior al que se estimaba. Por otra parte, el beta de Bancolombia ajustado se encuentra más cerca del beta sin ajustar debido a que la persistencia de este es significativa y, por lo tanto, el riesgo sistémico es mayor.

Gráfico 8

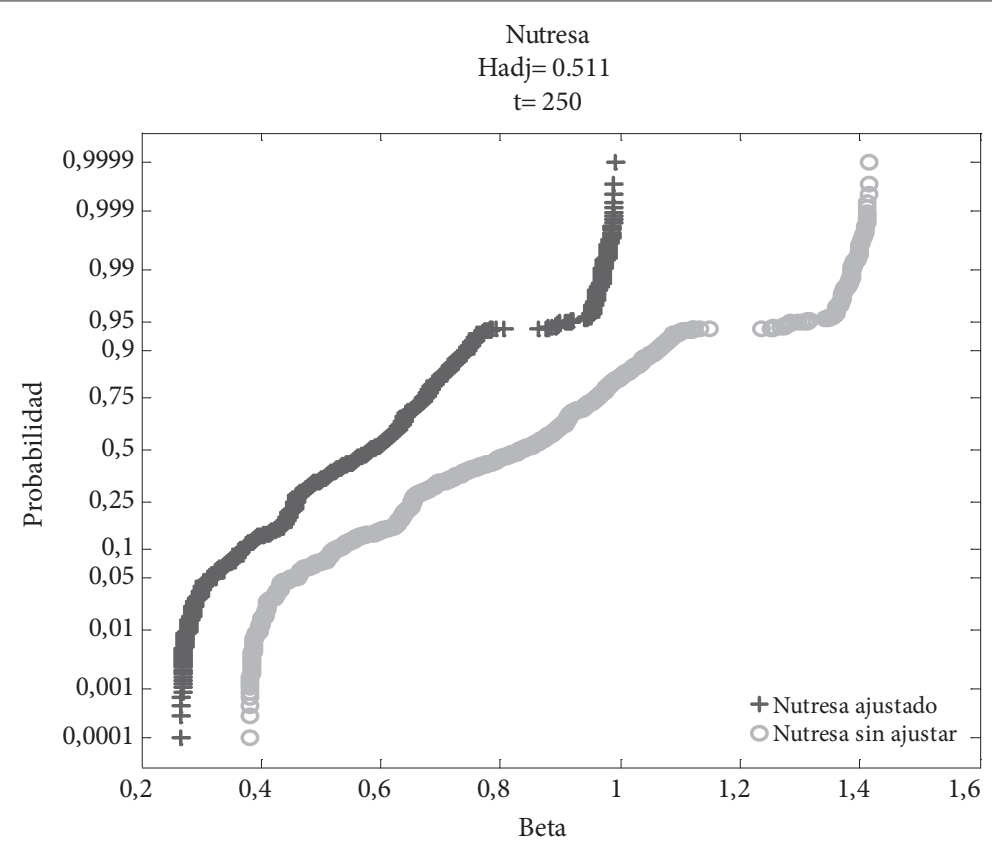




\section{Gráfico 9}

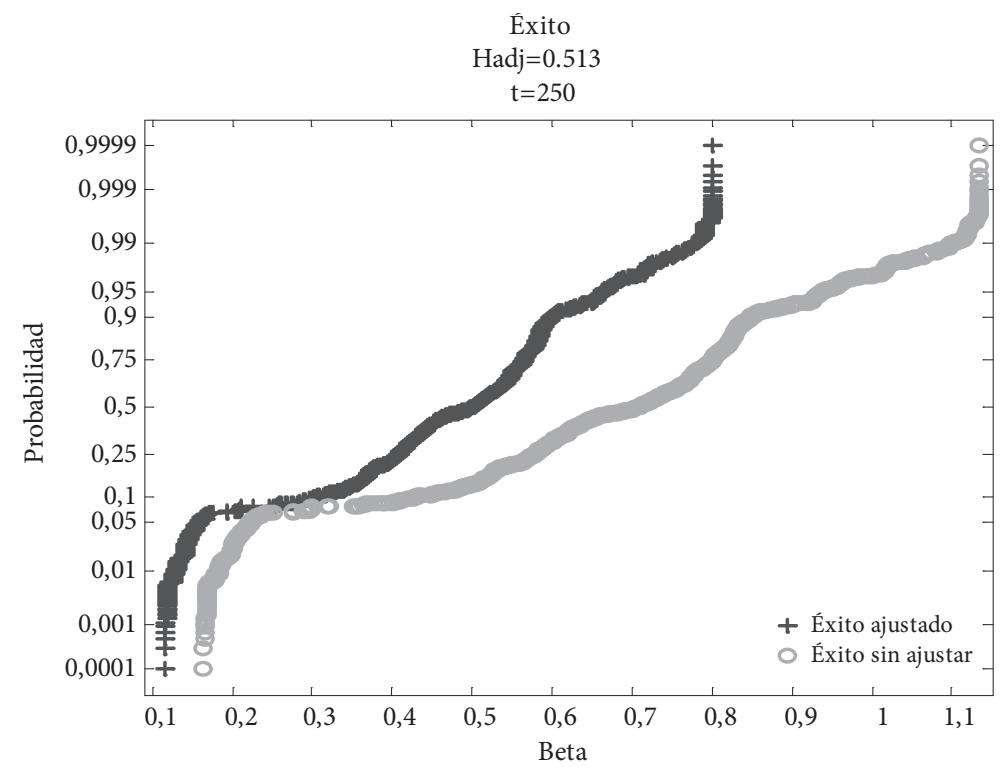

Fuente: Cálculos del autor

Gráfico 10

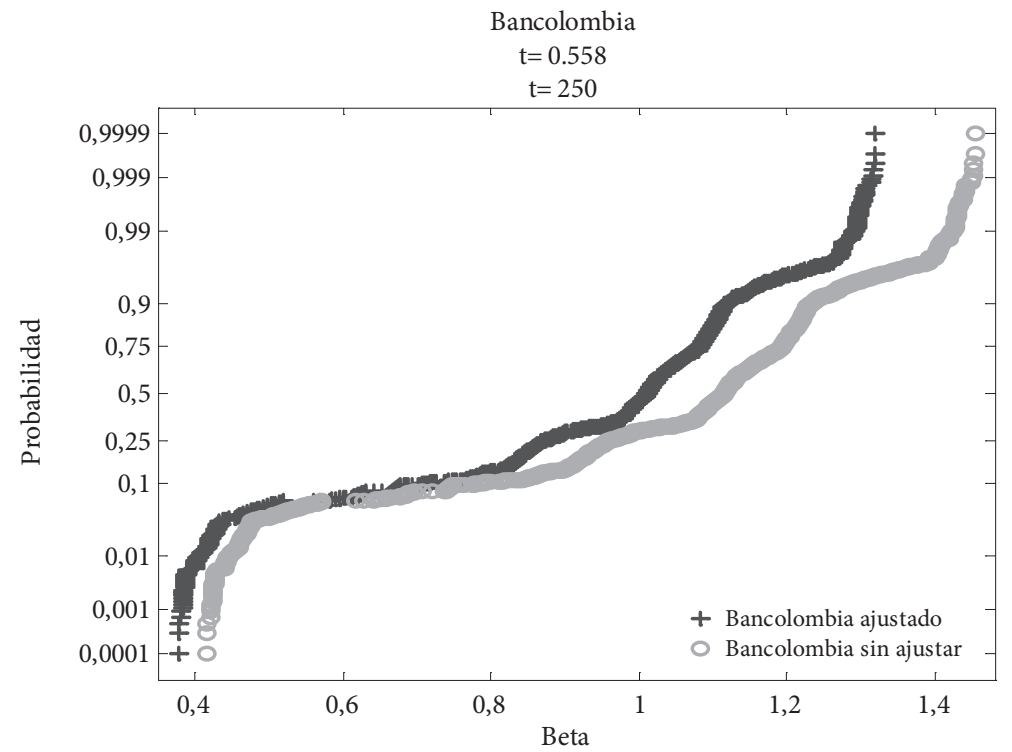

Fuente: Cálculos del autor 
Las diferencias en las distribuciones también se evidencian al comparar las principales estadísticas. Se corrobora que en los tres casos la media y desviación estándar de la distribución de los betas ajustados por dependencia de largo plazo es menor y que esta diferencia es mayor en las acciones de Nutresa y Éxito.

Cuadro 2. Estadísticas de la distribución de los betas estimados para un horizonte de inversión de un año (ajustados or dependencia de largo plazo y sin ajustar)

\begin{tabular}{|l|c|c|c|c|c|c|}
\hline \multirow{2}{*}{} & \multicolumn{2}{|c|}{ Nutresa } & \multicolumn{2}{c|}{ Éxito } & \multicolumn{2}{c|}{ Bancolombia } \\
\cline { 2 - 7 } & Sin ajustar & Ajustado & Sin ajustar & Ajustado & Sin ajustar & Ajustado \\
\hline Media & 0,82 & 0,57 & 0,67 & 0,47 & 1,06 & 0,96 \\
\hline Desviación estándar & 0,22 & 0,16 & 0,19 & 0,13 & 0,21 & 0,19 \\
\hline Asimetría & 0,36 & 0,36 & $-0,66$ & $-0,66$ & $-1,29$ & $-1,29$ \\
\hline Curtosis & 3,12 & 3,12 & 3,61 & 3,61 & 4,73 & 4,73 \\
\hline
\end{tabular}

Fuente: cálculos del autor.

Dado que los gráficos de probabilidad de los betas (Gráficos 8, 9 y 10) muestran que estos no se distribuyen como una normal ${ }^{20}$, se aplican dos pruebas no paramétricas $^{21}$ con el propósito de comparar las dos distribuciones. Por un lado, la prueba de Kolmogorov-Smirnov calcula la desviación de las funciones de distribución acumuladas de dos series de tiempo, donde la hipótesis nula es que las series provienen de la misma distribución continua. Adicionalmente, se aplica la prueba $U$ de Mann-Whitney, cuya hipótesis nula corresponde a que dos series de tiempo son muestras independientes pertenecientes a distribuciones idénticas, continuas y con medianas iguales. La hipótesis alternativa es que no tienen la misma mediana.

Según los resultados de estas pruebas para las distribuciones de los betas estimados para cada una de las acciones, con un $99 \%$ de confianza, se rechaza la hipótesis nula de que la distribución de los betas que incorporan la dependencia de largo plazo es igual a la que supone independencia.

20 Esto se corroboró con las pruebas estándar para probar normalidad, Jarque-Bera, Lilliefors y Kolmogorov-Smirnov. Para las tres pruebas se rechazó la hipótesis nula de normalidad.

21 La prueba de Kolmogorov-Smirnov compara la distribución empírica de dos muestras y el estadístico se calcula como la máxima diferencia absoluta entre las dos distribuciones. Dadas dos variables $X$ y $Y$ ordenadas de forma ascendente, la prueba $U$ de Mann-Whitney está definida como el número de veces que $Y$ precede a $X$. La metodología de cálculo se encuentra en detalle en Gibbons y Chakraborti (2003).

PP. $203-245 \cdot N .{ }^{\circ} 6 / 2011$ 
Cuadro 3

\begin{tabular}{|l|c|c|c|c|}
\hline \multicolumn{1}{|c|}{ Acción } & Estadístico Kolmogrov-Smirnof & P-val & Z del estadístico U Mann-Whitney & P-val \\
\hline Nutreza & 0,524 & 0 & $-54,87$ & 0 \\
\hline Éxito & 0,5876 & 0 & $-56,10$ & 0 \\
\hline Bancolombia & 0,3966 & 0 & $-34,28$ & 0 \\
\hline
\end{tabular}

Fuente: cálculos del autor.

Por otra parte, el ejercicio realizado para las acciones de JPMorgan, Walmart y Kellog's muestra que, contrario a los resultados para las acciones de Colombia, la distribución de los betas ajustados por dependencia de largo plazo se encuentra a la derecha de la que no incorpora este ajuste. Aunque solo la acción de Walmart muestra una distancia significativa de la hipótesis de independencia, el índice S\&P500 también muestra un comportamiento anti-persistente de similar magnitud, por lo que el beta ajustado de esta acción varía menos que en el caso de otras acciones.

\section{Gráfico 11}

JPMorgan
Hadj= 0.4896
$t=250$


Gráfico 12

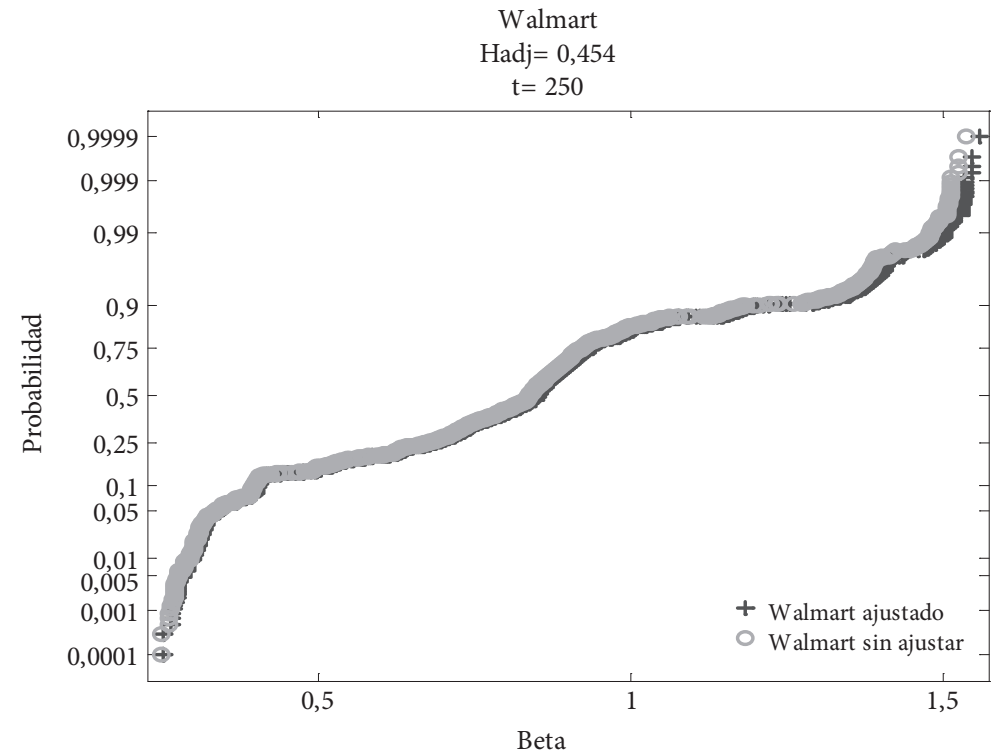

Fuente: Cálculos del autor

Gráfico 13

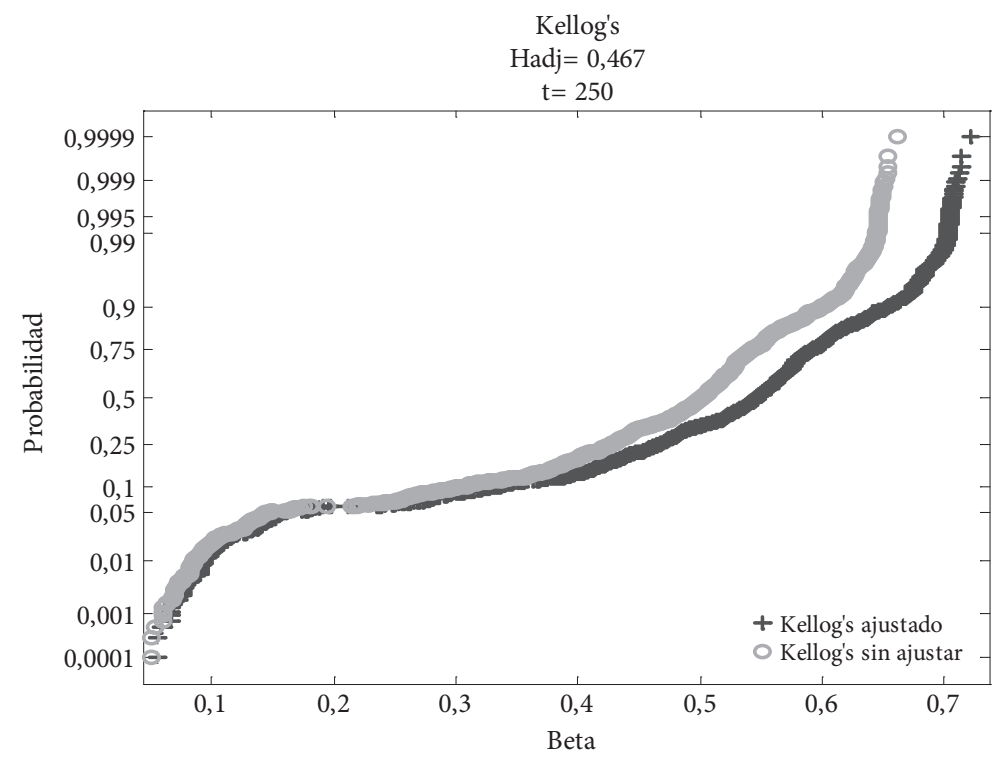

Fuente: Cálculos del autor.

PP. $203-245 \cdot$ N. $^{\circ} 6 / 2011$ 
Cuadro 4. Estadísticas de la distribución de los betas estimados para un horizonte de inversión de un año (ajustados por dependencia de largo plazo y sin ajustar)

\begin{tabular}{|l|c|c|c|c|c|c|}
\hline \multirow{2}{*}{} & \multicolumn{2}{|c|}{ JPMorgan } & \multicolumn{2}{c|}{ Walmart } & \multicolumn{2}{c|}{ Kellogs } \\
\cline { 2 - 7 } & Sin ajustar & Ajustado & Sin ajustar & Ajustado & Sin ajustar & Ajustado \\
\hline Media & 1,42 & 1,73 & 0,81 & 0,83 & 0,47 & 0,51 \\
\hline Desviación estándar & 0,28 & 0,34 & 0,27 & 0,28 & 0,12 & 0,13 \\
\hline Asimetría & 0,79 & 0,79 & 0,23 & 0,23 & $-1,47$ & $-1,41$ \\
\hline Curtosis & 4,40 & 4,40 & 3,12 & 3,12 & 4,97 & 4,97 \\
\hline
\end{tabular}

Fuente: cálculos del autor.

El Cuadro 4 confirma que la media y desviación estándar de la distribución de los betas ajustados por dependencia de largo plazo es mayor y que esta diferencia es mayor en las acciones de JPMorgan y Kellog's.

No obstante, es importante anotar que la distribución del beta de la acción de Walmart con el ajuste es muy similar a la que no lo tiene, lo cual se explica porque el exponente de Hurst de la acción (0.454) es cercano al exponente del índice (0.451). Con el fin de comprobar estadísticamente las diferencias en estas distribuciones se realizaron las mismas pruebas no paramétricas que se realizaron para las acciones colombianas. Los resultados del Cuadro 5 muestran que con el 99\% de confianza se rechaza la hipótesis de igualdad de las distribuciones de los betas ajustados y sin ajustar.

Cuadro 5

\begin{tabular}{|l|c|c|c|c|}
\hline \multicolumn{1}{|c|}{ Acción } & Estadístico Kolmogrov-Smirnof & P-val & Z del estadístico U Mann-Whitney & P-val \\
\hline JPMorgan & 0,4422 & 0 & 46,87 & 0 \\
\hline Walmart & 0,0598 & 0 & 3,37 & 0,0008 \\
\hline Kellogs & 0,2806 & 0 & 23,02 & 0 \\
\hline
\end{tabular}

Fuente: cálculos del autor.

El único caso en que la neutralidad en el horizonte de inversión se mantiene en presencia de dependencia de largo plazo es cuando la persistencia del activo es igual a la del portafolio de mercado. Como se mencionó, la acción de Walmart tiene un exponente de Hurst muy cercano al del mercado, pero la diferencia resulta en un beta significativamente diferente según los estadísticos utilizados. 
Aunque Peters (1977) afirma que es poco probable encontrar una acción con un exponente de Hurst igual al del portafolio de mercado, la acción de Procter \& Gamble tiene esa característica. Lo anterior, resulta en que la distribución de los betas estimados, suponiendo independencia, es igual a la distribución con el ajuste por dependencia, lo cual es confirmado con los resultados de las pruebas paramétricas del Cuadro 7, que no rechazan la hipótesis de igualdad de las distribuciones.

Gráfico 14

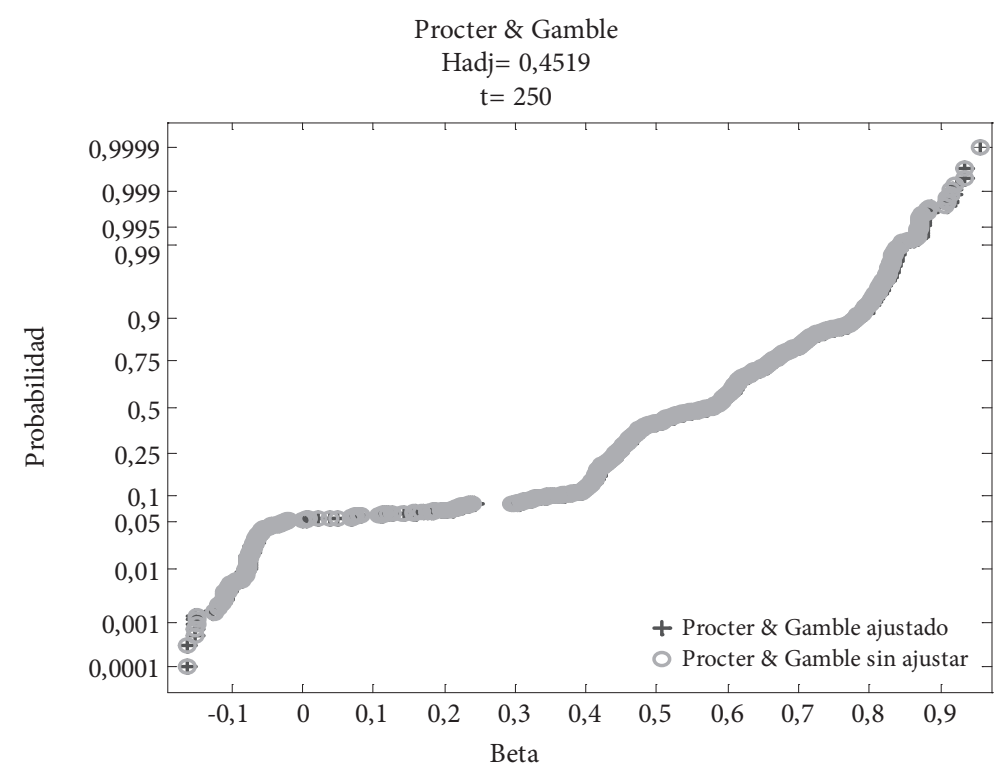

Fuente: Cálculos del autor.

Cuadro 6. Estadísticas de la distribución de los betas estimados para un horizonte de inversión de un año (ajustados por dependencia de largo plazo y sin ajustar)

\begin{tabular}{|l|c|c|}
\hline \multirow{2}{*}{} & \multicolumn{2}{|c|}{ P\&G } \\
\cline { 2 - 3 } & Sin ajustar & Ajustado \\
\hline Media & 0,53 & 0,54 \\
\hline Desviación estándar & 0,20 & 0,21 \\
\hline Asimetría & $-1,20$ & $-1,20$ \\
\hline Curtosis & 4,91 & 4,91 \\
\hline
\end{tabular}

Fuente: Cálculos del autor. 
Cuadro 7

\begin{tabular}{|c|c|c|c|c|}
\hline Acción & Estadístico Kolmogrov-Smirnof & P-val & Z del estadístico U Mann-Whitney & P-val \\
\hline P\&G & 0,0134 & 0,76 & 0,62 & 0,53 \\
\hline
\end{tabular}

Fuente: Cálculos del autor.

Asimismo, las diferencias en los betas se reflejan en los retornos esperados de las acciones. Para las acciones de Nutresa, Éxito y Bancolombia los retornos esperados promedio $^{22}$ con el ajuste por dependencia de largo plazo son 111, 90 y 43 p.b., inferiores a los retornos esperados que no incorporan el ajuste. Para las acciones de JPMorgan, Walmart y Kellog's el cambio en el retorno esperado es inferior al de las acciones colombianas, en promedio los retornos caen 73, 2 y 9 p.b. , cuando se incorpora el ajuste por dependencia de largo plazo.

Cuadro 8. Promedio del retorno esperado anual

\begin{tabular}{|c|l|c|c|c|}
\hline \multicolumn{1}{|c|}{ Activo } & & Ajustado & Sin ajustar & Ajuste en p.b. \\
\hline \multirow{4}{*}{ Colombia } & Nutresa & $18,3 \%$ & $19,4 \%$ & -111 \\
\cline { 2 - 5 } & Éxito & $17,9 \%$ & $18,8 \%$ & -90 \\
\cline { 2 - 5 } & Bancolombia & $19,9 \%$ & $20,3 \%$ & -43 \\
\hline \multirow{3}{*}{ Estados Unidos } & JPMorgan & $-1,5$ & $-0,7$ & -73 \\
\cline { 2 - 5 } & Walmart & $1,0 \%$ & $1,0 \%$ & -2 \\
\cline { 2 - 5 } & Kellogs & $1,6 \%$ & $1,7 \%$ & -9 \\
\hline
\end{tabular}

Fuente: cálculos del autor.

De los resultados obtenidos se destaca un hecho interesante que contradice una de las creencias más populares en las finanzas: que los retornos promedio de los instrumentos de renta fija es inferior al de las acciones. Este hecho, destacado también por León y Reveiz (2010), Valdés (2010) y Arnott (2010), se evidencia en el comportamiento de la acción de JPMorgan, que presenta un retorno promedio esperado negativo al utilizar una serie de tiempo considerablemente extensa.

22 Promedio simple de los datos diarios de retornos esperados anuales de la muestra analizada. 
Gráfico 15

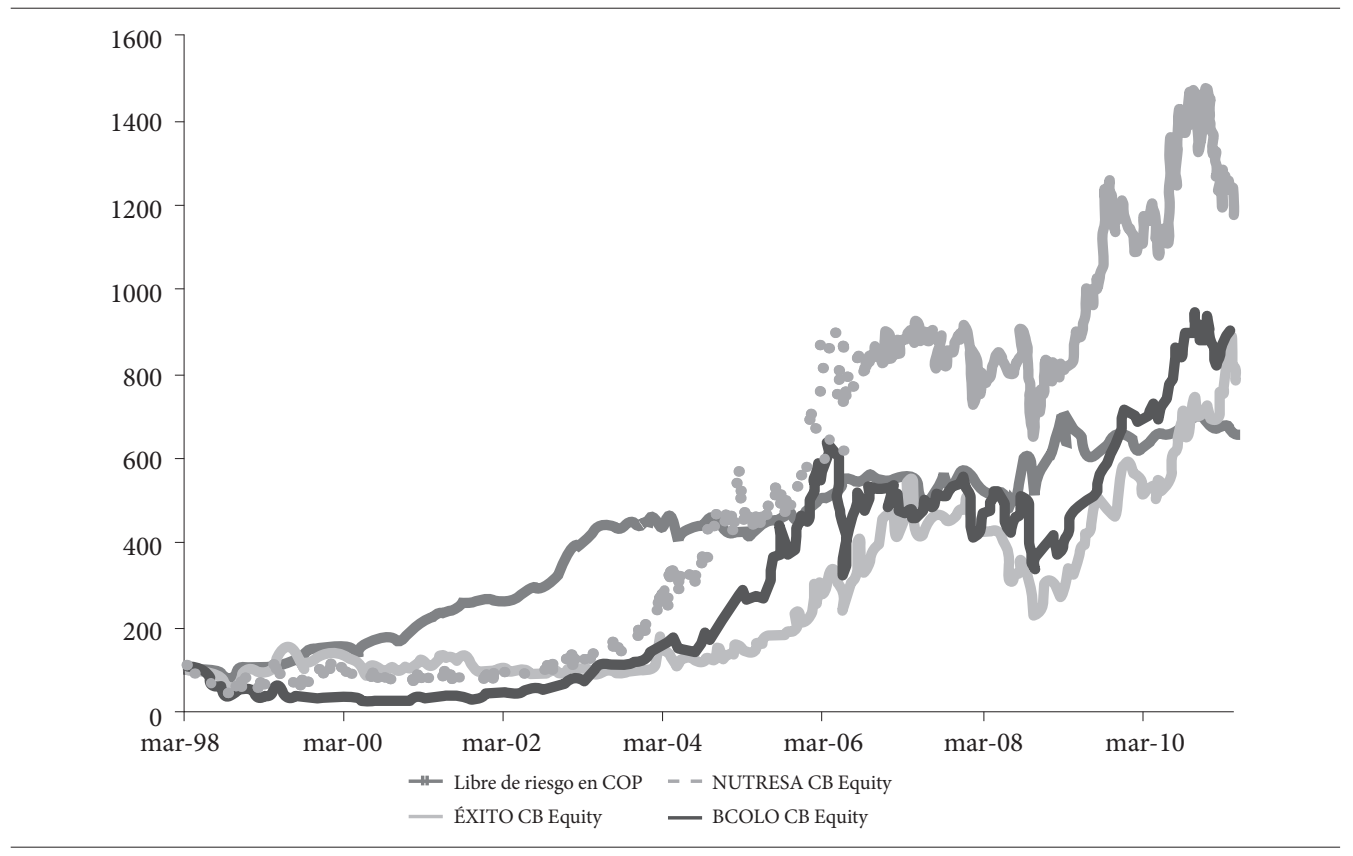

Gráfico 16

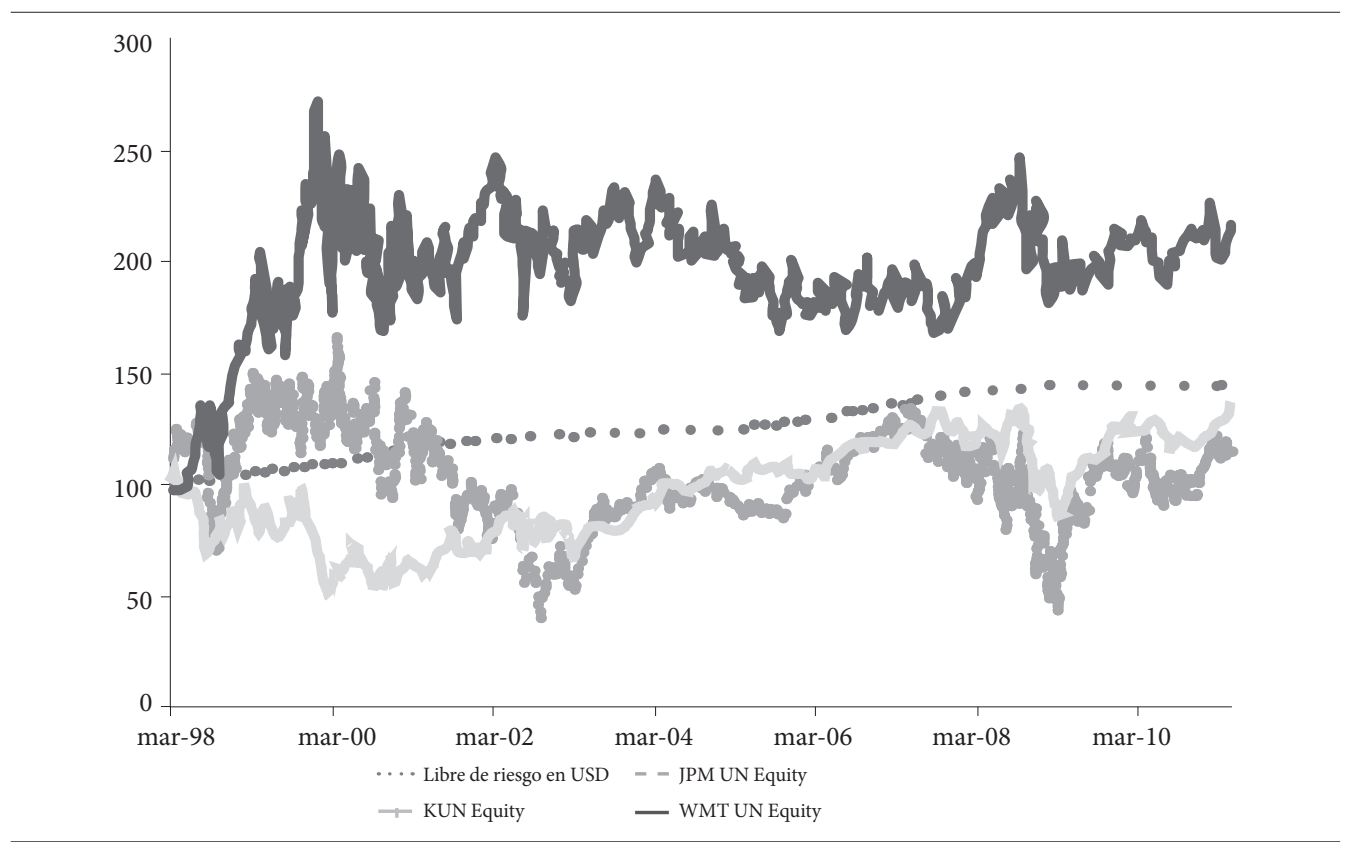

Fuente: Cálculos del autor

PP. $203-245$ • N. ${ }^{\circ} 6 / 2011$ 


\section{Conclusiones}

Además de ser uno de los pilares de la teoría financiera moderna, el CAPM es comúnmente utilizado en la valoración de empresas y en otras aplicaciones en el mercado de capitales. En la práctica se ignora que el modelo fue diseñado para un solo período de inversión, y se utiliza para períodos más extensos suponiendo que existe neutralidad del horizonte de inversión. Este documento, a partir de los hallazgos de Peters (1989 y 1992), Mandelbrot (1972), León y Vivas (2010), entre otros, respecto de la dependencia de largo plazo de las series de tiempo financieras, mostró que este fenómeno invalida el supuesto de neutralidad en el horizonte de tiempo en el CAPM y, en consecuencia, cambia sus resultados.

La no neutralidad en el horizonte de inversión, causada por la presencia de dependencia de largo plazo en los activos, implica que la información del riesgo contenida en un período corto difiere de la de un plazo mayor. Debido a esto, las decisiones de inversión basadas en la relación riesgo-retorno y los resultados del CAPM cambian al escalar la volatilidad con el exponente de Hurst ajustado. Basado en Greene y Fieltz (1980), se demostró cómo cambia la formulación del CAPM al escalar las varianzas del mercado y del activo, así como su covarianza, utilizando el exponente de Hurst estimado para cada uno de ellos.

Con esta metodología se compararon los betas y los retornos esperados del CAPM para seis acciones, tres pertenecientes al mercado colombiano y tres del mercado estadounidense. Se encontró que los betas de las acciones de Nutresa, Éxito y Bancolombia, que incorporan dependencia de largo plazo, son menores que los que no tienen el ajuste; esto se explica porque el exponente de Hurst ajustado del mercado (IGBC) es mayor que el de las acciones. Adicionalmente, se realizaron pruebas estadísticas no paramétricas, las cuales resultaron en el rechazo de la hipótesis nula de igualdad de las distribuciones de los betas de las acciones analizadas con y sin el ajuste.

Por otra parte, el ejercicio realizado para las acciones de JPMorgan, Walmart y Kellog's muestra que, contrario a los resultados para las acciones de Colombia, la distribución de los betas ajustados por dependencia de largo plazo se encuentra a la derecha de la que no incorpora este ajuste, lo cual se explica porque el exponente de Hurst ajustado del mercado (S\&P500) es menor que el de las acciones. Igualmente, se realizaron pruebas estadísticas no paramétricas, las cuales resultaron en el rechazo de la hipótesis nula de igualdad de las distribuciones de los betas de las acciones con y sin el ajuste. 
Asimismo, las diferencias en los betas se reflejan en los retornos esperados de las acciones. Para las acciones colombianas analizadas los retornos esperados con el ajuste por dependencia de largo plazo caen en promedio 80 p.b. Las acciones estadounidenses muestran un cambio en los retornos esperado inferior al de las acciones colombianas, en promedio estos caen 28 p.b.

Los resultados de este trabajo son útiles, pues los trabajos futuros, que se basen en el CAPM pueden conseguir capturar el efecto de la dependencia de largo plazo a través de la metodología mostrada. Adicionalmente, este documento puede resultar de utilidad para los participantes del mercado de capitales local, ya que es una forma fácil e intuitiva de realizar el ajuste por dependencia de largo plazo en el CAPM.

En cuanto a las extensiones, este trabajo puede ser retomado en el futuro para incluir la multifractalidad en el CAPM. Adicionalmente, se considera explorar otras metodologías para cuantificar la dependencia de largo plazo e incorporarlas en el modelo de valoración.

\section{Bibliografía}

Amenc, N. y Le Sourd, V. (2003). Portfolio Theory and Performance Analysis; capítulo 4, Chichester. JohnWiley \& Sons.

Anis, A.A. y Lloyd, E.H. (1976). "The Expected Value of the Adjusted Rescaled Hurst Range of Independent Normal Summands", Biometrika, n. ${ }^{\circ}$ 1, vol.63.

Arnnott, R. (2009). “Bonds: Why Bother”, Journal of Indexes, mayo/junio.

Black, F. (1972). "Capital Market Equilibrium with Restricted Borrowing"; Journal of Business, n. ${ }^{\circ} 45$, pp. 444-455.

Black, F., y Scholes, M. (1973). “The Pricing of Options and Corporate Liabilities”. Journal of Political Economy, vol. 81, n. ${ }^{\circ} 3$.

Bollerslev, T. (1986). "Generalized Autoregressive Conditional Heteroskedasticity". Journal of Econometrics, n. $^{\circ} 31$.

Brennan, M. (1970). "Taxes, Market Valuation and Corporate Financial Policy”, National Tax Journal, n. ${ }^{\circ}$ 25, pp. 417-427.

Campbell, J.; Lo, A.; MacKinlay, C. (1997). The Econometrics of Financial Markets: cap. 5. Pricenton, NJ. Princeton University Press.

PP. 203-245 • N. ${ }^{\circ} 6 / 2011$ 
Couillard, M. y Davison, M. (2005) “A Comment on Measuring the Hurst Exponent of Financial Time Series”, Physica A, n. 348.

Cuthbertson, K. y Nitzsche, D. (2004). Quantitative Financial Economics. Chichester. JohnWiley \& Sons.

Editorial. (2005). The Rough Guide. Wilmott Magazine, mar/abr.

Engle, R. (1982). “Autoregressive Conditional Heteroscedasticity with Estimates of the Variance of United Kingdom Inflation”. Econometrica, vol. 50, issue 4.

Ellis, C. (2007). “The Sampling Properties of Hurst Exponent Estimates", Physica A, n. ${ }^{\circ}$ 375.

Fama E.F. (1965). "The Behavior of Stock-Market Prices"; The Journal of Business, vol. 38, n. $^{\circ} 1$.

Fama E.F. (1969). "Efficient Capital Markets: A Review of Theory and Empirical Work"; The Journal of Finance, vol. 25, n. ${ }^{\circ}$ 2, Papers and Proceedings of the Twenty-Eighth Annual Meeting of the American Finance Association New York.

Fama E.F. (1973). "A Note on the Market Model and the Two-Parameter Model”; The Journal of Finance, vol. 28, n. ${ }^{\circ} 5$.

Fama E.F. (1963). "Mandelbrot and the Stable Paretian Hypothesis"; The Journal of Business, vol. 36, n. $^{\circ} 4$.

Fama E.F. (1968) "Risk, Return and Equilibrium: Some Clarifying Comments"; The Journal of Finance, vol. 23, n. ${ }^{\circ} 1$.

Gibbons, J. and Chakraborti, S. (2003). Nonparametric Statistical Inference, capítulo 6, New York. Marcel Dekker.

Greene, M.T. y Fielitz, B.D. (1979) “The Effect of Long-Term Dependence on Risk-Return Models of Common Stocks"; Operations Research, vol. 27, n. 5.

Greene, M.T. y Fielitz, B.D. (1980). “Long-Term Dependence and Least Squares Regression in Investment Analysis"; Management Science, vol. 26, n. ${ }^{\circ} 10$. 
Holton, G.A. (1992). "Time: The second dimension of risk". Financial Analysts Journal; n. ${ }^{\circ} 48$, vol. 6 .

León, C. y Vivas, F. (2010) “Dependencia de Largo Plazo y la Regla de la Raíz del Tiempo para Escalar la Volatilidad en el Mercado Colombiano”, Borradores de Economía, n. ${ }^{\circ} 603$, Banco de la República.

León, C. y Reveiz, A. (2010). "Portfolio Optimization and Long-Term Dependence", Borradores de Economía, n. ${ }^{\circ}$ 648, Banco de la República.

León, C. y Reveiz, A. (2011). "Montecarlo Simulation of Long-Term Dependent Processes: a primer”, Borradores de Economía, n. ${ }^{\circ} 622$, Banco de la República.

León, C. (2009). "Una Aproximación Teórica a la Superficie de Volatilidad del Mercado Colombiano a través del Modelo de Difusión con Saltos”, Borradores de Economía, n. ${ }^{\circ}$ 570, Banco de la República.

Litterman, B. (2003). “Modern Investment Management, an Equilibrium Aproach”. Hoboken, NJ. John Wiley and Sons. cap 4.

Lo, A.W. (1991). "Long-Term Memory in Stock Market Prices”, Econometrica, vol. 59, n. ${ }^{\circ} 5$.

Mandelbrot, B. (1965). "Une Classe de Processus Stochastiques Homothétiques à Soi. Application à la Loi Climatologique de H. E. Hurst", Comptes Rendus Academie de Science de Paris, vol. 260.

Mandelbrot, B. (1972). "Statistical Methodology for Nonperiodic Cycles: from the covariance to the R/S Analysis", Annal of Economic and Social Measuremente, NBER, vol. 1, n. $^{\circ} 3$.

Mandelbrot B. y Hudson, R.L. (2004). The (Mis)Behavior of Markets, New York. Basic Books.

Marín, J. y Rubio, G. (2001). Economía Financiera, capítulo 6, 7 y 19. Barcelona. Bosch Editors.

Markowitz, H.M. (1959), Portfolio Selection: Efficient Diversification of Investments, New York. John Wiley \& Sons. 
Mathworks (2009) Statistics Toolbox User's Guide. The Mathworks. marzo.

McLeod, A. and Hipel, K. (1978). "Preservation of the Rescaled Adjusted Range, A Preassesment of the Hurst Phenomenon”. Water Resources Research.

Merkens, O. (2007). "Value at Risk and Self-Similarity". Numerical Methods for Finance. (Edited by: Miller, J., Edelman, D., Appleby, J.), Chapman 6 Hall/CRC Financial Mathematics Series.

Merton, R.C. (1976). “Option Pricing when Underlying Stock Returns are Discontinuous”. Journal of Financial Economics.

Nielsen, L. (1999). Pricing and Hedging of Derivative Securities, Oxford University Press.

Peters, E.E. (1989). "Fractal Structure In The Capital Markets"; Financial Analysts Journal, n. ${ }^{\circ} 45$, vol. 4.

Peters, E.E. (1992). "R/S Analysis using logarithmic returns"; Financial Analysts Journal; n. ${ }^{\circ} 48$, vol. 6 .

Peters, E.E. (1994). Fractal Market Analysis. New York. John Wiley \& Sons.

Peterson, I. , (1998) The Jungles of Randomness, A mathematical safari. New York. John Wiley \& Sons, cap 8.

Ross, S., Westerfield, R., Jaffe, J. (1998). Finanzas Corporativas. México. McGraw Hill.

Shojai, S. y Feiger, G. (2009). "Economists' hubris-the case of asset pricing”. Journal of Financial Transformation.

Sornette, D. (2003).Why Stock Markets Crash, Princeton, NJ. Princeton University Press.

Valdés, S. (2010). “Acciones, Plazo de Inversión y Multifondos”, Estudios Públicos, n. 117, Chile, Centro de Estudios Públicos. 


\section{Anexo 1. Exponente de Hurst y exponente de Hurst ajustado}

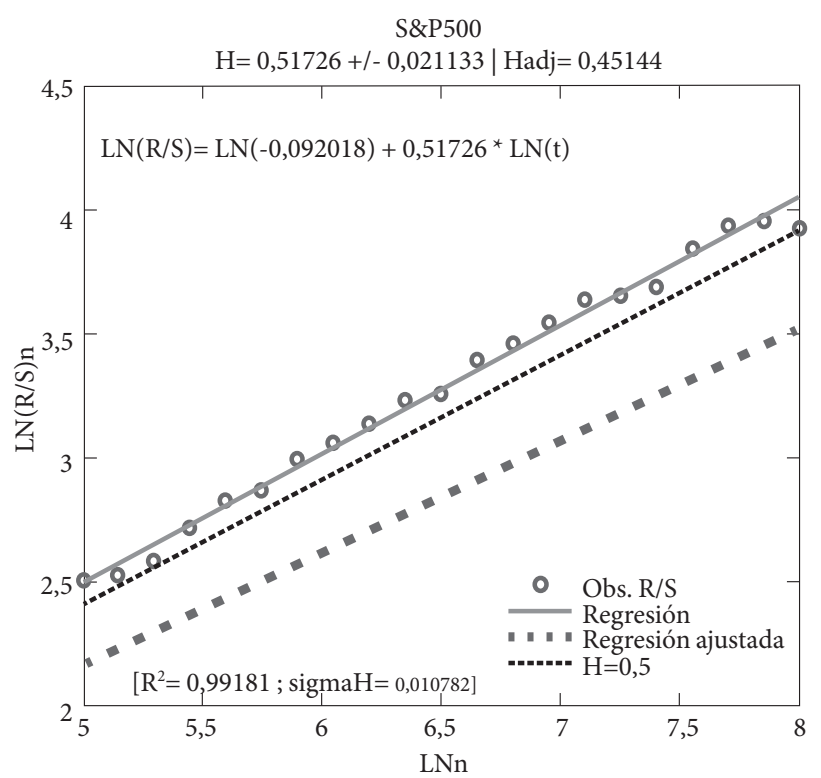

Fuente: Cálculos del autor.

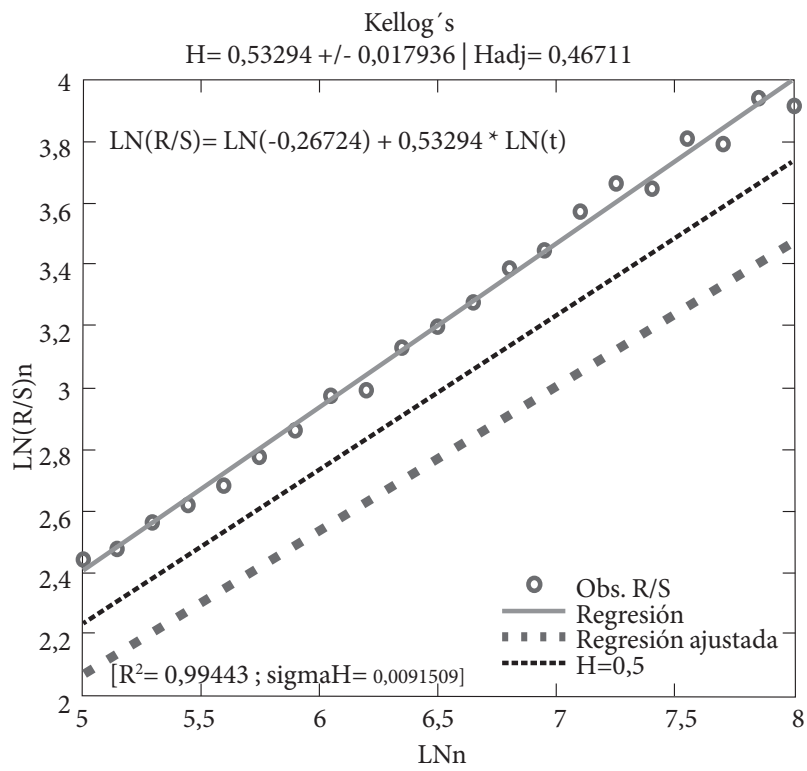

Fuente: Cálculos del autor. 
Walmart

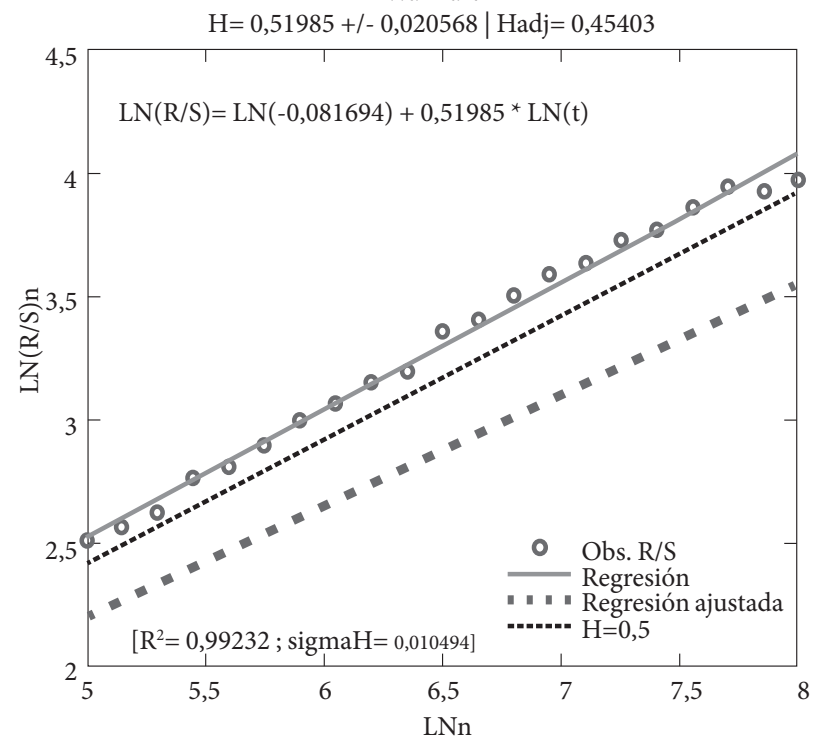

Fuente: Cálculos del autor.

JPMorgan

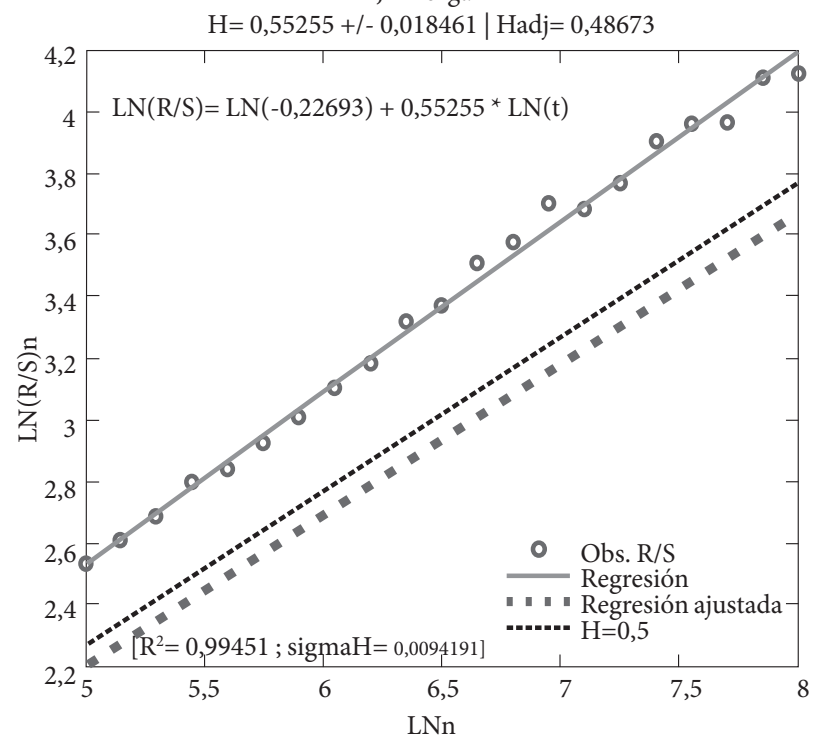

Fuente: Cálculos del autor. 